\title{
Random Graphs and the Strong Convergence of Bootstrap Means
}

\author{
SÁNDOR CSÖRGÖ1,2† and WEI BIAO WU ${ }^{1}$ \\ ${ }^{1}$ Department of Statistics, University of Michigan, \\ 4062 Frieze Building, Ann Arbor, MI 48109-1285, USA \\ (e-mail: scsorgo@umich.edu, wbwu@umich.edu) \\ 2 Bolyai Institute, University of Szeged, \\ Aradi vértanúk tere 1, H-6720 Szeged, Hungary \\ (e-mail: csorgo@math.u-szeged.hu)
}

Received 16 October 1998; revised 31 May 1999

We consider graphs $G_{n}$ generated by multisets $\mathscr{I}_{n}$ with $n$ random integers as elements, such that vertices of $G_{n}$ are connected by edges if the elements of $\mathscr{I}_{n}$ that the vertices represent are the same, and prove asymptotic results on the sparsity of edges connecting the different subgraphs $G_{n}$ of the random graph generated by $\cup_{n=1}^{\infty} \mathscr{J}_{n}$. These results are of independent interest and, for two models of the bootstrap, we also use them here to link almost sure and complete convergence of the corresponding bootstrap means and averages of related randomly chosen subsequences of a sequence of independent and identically distributed random variables with a finite mean. Complete convergence of these means and averages is then characterized in terms of a relationship between a moment condition on the bootstrapped sequence and the bootstrap sample size. While we also obtain new sufficient conditions for the almost sure convergence of bootstrap means, the approach taken here yields the first necessary conditions.

\section{Introduction}

Let $\left\{X_{n}\right\}_{n=0}^{\infty}$ be a sequence of independent and identically distributed random variables with a finite mean $\mu:=\mathbb{E}(X)$, where $X=X_{0}$. Given the sample $X_{0}, \ldots, X_{n-1}$ for some sample size $n \in \mathbb{N}$, where $\mathbb{N}$ is the set of natural numbers, Efron's [11] ordinary bootstrap sample $Y_{n, 0}, \ldots, Y_{n, m_{n}-1}$ of size $m_{n} \in \mathbb{N}$ results from resampling $m_{n}$ times the sequence $X_{0}, \ldots, X_{n-1}$ with replacement such that at each stage any one element has probability $1 / n$ of being selected; in other words, given $X_{0}, \ldots, X_{n-1}$, the random variables $Y_{n, 0}, \ldots, Y_{n, m_{n}-1}$ 
are conditionally independent and their common distribution is the sample distribution. The bootstrap mean, then, is

$$
\mu_{n}\left(m_{n}\right):=m_{n}^{-1} \sum_{j=0}^{m_{n}-1} Y_{n, j}=m_{n}^{-1} \sum_{j=0}^{m_{n}-1} X_{Z_{n, j}},
$$

where $\left\{Z_{n, 0}, \ldots, Z_{n, m_{n}-1}\right\}_{n=1}^{\infty}$ is a triangular array of row-wise independent random variables, given on the same probability space $(\Omega, \mathscr{A}, \mathbb{P})$ where the sequence $\left\{X_{n}\right\}_{n=0}^{\infty}$ is defined, such that the sequence $\left\{X_{n}\right\}_{n=0}^{\infty}$ and the whole array $\left\{Z_{n, 0}, \ldots, Z_{n, m_{n}-1}\right\}_{n=1}^{\infty}$ are independent and $\mathbb{P}\left\{Z_{n, j}=k\right\}=1 / n, k=0, \ldots, n-1$, for every $j \in\left\{0, \ldots, m_{n}-1\right\}$ and $n \in \mathbb{N}$. Another suggestive form is $\mu_{n}\left(m_{n}\right)=\sum_{k=0}^{n-1} w_{n, k}\left(m_{n}\right) X_{k}$, where the sequence of random weight vectors $\mathbf{w}_{n}\left(m_{n}\right)=\left(w_{n, 0}\left(m_{n}\right), \ldots, w_{n, n-1}\left(m_{n}\right)\right)$ of frequencies $w_{n, k}=m_{n}^{-1} \sum_{j=0}^{m_{n}-1} I\left(Z_{n, j}=k\right)$ is independent of the basic sequence and $m_{n} \mathbf{w}_{n}\left(m_{n}\right)$ has the $\operatorname{Multinomial}\left(m_{n} ; 1 / n, \ldots, 1 / n\right)$ distribution, where $I(A)$ denotes the indicator function of an event $A \in \mathscr{A}$.

The weak law of large numbers for the bootstrap mean, that is, when $\mu_{n}\left(m_{n}\right) \rightarrow \mu$ in probability, holds whenever $m_{n} \rightarrow \infty$. This goes back to [6]; there is a simpler proof in [3], while the simplest proof is in [10] ( $c f$. the third part of the theorem in [10]). (Here and throughout, convergence and order relations are meant as $n \rightarrow \infty$ unless otherwise specified.) Concerning the problem of almost sure (a.s.) or strong convergence of $\mu_{n}\left(m_{n}\right)$ to $\mu$, a matter of style should first be clarified. Let $\mathbb{P}_{\mathbf{X}}(H):=\mathbb{P}\{\mathbf{X} \in H\}, H \in \mathscr{B}^{\infty}$, be the distribution of the sequence $\mathbf{X}:=\left\{X_{n}\right\}_{n=0}^{\infty}$ over $\left(\mathbb{R}^{\infty}, \mathscr{B}^{\infty}\right)$, where $\mathscr{B}$ is the class of Borel sets in the real line $\mathbb{R}$. Numerous papers state strong laws in the conditional form, which for $\mu_{n}\left(m_{n}\right)$ is some verbal equivalent of the equation $\mathbb{P}_{\mathbf{X}}(B)=1$, where $B=\left\{\mathbf{x} \in \mathbb{R}^{\infty}: \mathbb{P}\{A \mid \mathbf{X}=\mathbf{x}\}=1\right\}$ for the event $A=\left\{\mu_{n}\left(m_{n}\right) \rightarrow \mu\right\}$. Since there exists a unique Borel function $p_{A}: \mathbb{R}^{\infty} \mapsto[0,1]$ for which $p_{A}(\mathbf{X})$ is a version of $\mathbb{P}\{A \mid \mathbf{X}\}$ and $\mathbb{P}\{A \mid \mathbf{X}=\mathbf{x}\}$ is understood as $p_{A}(\mathbf{x})$ for each $\mathbf{x} \in \mathbb{R}^{\infty}$, we have $\{\omega \in \Omega: \mathbf{X}(\omega) \in B\}=\{\omega \in$ $\left.\Omega: p_{A}(\mathbf{X}(\omega))=1\right\}=\{\mathbb{P}\{A \mid \mathbf{X}\}=1\}$ for this version, and hence it is easy to see, in fact for any event $A \in \mathscr{A}$, that the three statements $\mathbb{P}\{\mathbb{P}\{A \mid \mathbf{X}\}=1\}=1, \mathbb{P}_{\mathbf{X}}\left(\left\{\mathbf{x} \in \mathbb{R}^{\infty}: \mathbb{P}\{A \mid \mathbf{X}=\right.\right.$ $\mathbf{x}\}=1\})=1$ and $\mathbb{P}\{A\}=1$ are equivalent. Thus, although this is not always clear in the literature, conditional and unconditional bootstrap strong laws are one and the same.

The problem of the strong law of large numbers for the bootstrap mean $\mu_{n}\left(m_{n}\right)$ is difficult. It is intuitively clear that the faster the bootstrap sample size $m_{n}$ grows with the sample size $n$, the better is the chance of having $\mu_{n}\left(m_{n}\right) \stackrel{\text { a.s. }}{\longrightarrow} \mu$ because then $\mu_{n}\left(m_{n}\right)$ will probably be closer to the sample mean

$$
\bar{\mu}_{n}:=n^{-1} \sum_{j=0}^{n-1} X_{j} .
$$

Theorem 4.1 below shows that the condition $\liminf _{n \rightarrow \infty}[\log n] / m_{n}=0$ is in fact necessary for any bootstrap strong law in two reasonable models of the bootstrap if $X$ is nondegenerate.

Once $m_{n} \rightarrow \infty$ faster than $\log n$, it turns out that the slower this happens, the stronger the moment conditions beyond $\mathbb{E}(|X|)<\infty$ needed to compensate. The relationship is intricate, and the first necessary conditions are obtained in the present paper. Concerning sufficient conditions, Athreya [2] achieved the first result and some improvements were obtained in [3]. (Theorem 3.1 in [17], somewhat weaker than Theorem 2 in [3], is proved 
by a different method.) $\mathrm{Hu}$ [16] and Csörgő [10] aimed at some further improvements. However, it was pointed out by Arenal-Gutiérrez, Matrán and Cuesta-Albertos [1] that the second part of the proof for the first two statements of the theorem in [10] is in error. It will follow from Theorem 4.2 below that part of the statements in [10] and also of the statements in [16] are themselves incorrect. One of the best results to date is in [1]: if $\mathbb{E}\left(|X|^{\alpha}\right)<\infty$ and $\left[n^{1 / \alpha} \log n\right] / m_{n} \rightarrow 0$ for some $\alpha \geqslant 1$, then $\mu_{n}\left(m_{n}\right) \stackrel{\text { a.s. }}{\longrightarrow} \mu$. A slight improvement of this result, requiring only that $\liminf _{n \rightarrow \infty} m_{n} /\left[n^{1 / \alpha} \log n\right]>0$, and a version with a logarithmic factor in the moment condition for $X$ rather than in the growth condition for $m_{n}$, is in Theorem 4.4 below. This version allows the naive bootstrap, in which the bootstrap sample size is the same as the sample size at each step: $m_{n} \equiv n$. Previously, Mikosch [20] obtained comparable results. While the direct technique in [1] allows bootstrap strong laws for dependent variables as well (even the ergodic theorem may be bootstrapped), the conditional arguments in [20] yield Marcinkiewicz-Zygmundtype rates when $\mathbb{E}\left(|X|^{\alpha}\right)<\infty$ for some $\alpha \in[1,2]$, with $\alpha=2$ being the limitation of this method. (In fact, Mikosch [20] obtains some strong laws for $\mu_{n}\left(m_{n}\right)-\bar{\mu}_{n}$ even when $\alpha \in(0,1)$, so that $\bar{\mu}_{n}$ diverges a.s.; we do not deal with such laws, or with rates, here.)

We emphasize that all results mentioned so far are universal for $\mu_{n}\left(m_{n}\right)$ in that they hold for every possible joint distribution of the row vectors $\mathbf{Z}_{n}\left(m_{n}\right)=\left(Z_{n, 0}, \ldots, Z_{n, m_{n}-1}\right)$ of the triangular array $\left\{\mathbf{Z}_{n}\left(m_{n}\right)\right\}_{n=1}^{\infty}$ as long as the latter is independent of $\left\{X_{n}\right\}_{n=0}^{\infty}$. Since the statistician usually analyses a single given data set, practical ideas about the bootstrap do not specify such joint distributions. However, specifications become unavoidable when aiming at necessary conditions for $\mu_{n}\left(m_{n}\right) \stackrel{\text { a.s. }}{\longrightarrow} \mu$.

In this paper we consider two special models for the bootstrap. The first is a triangular array situation with independent rows, in which the array $\left\{Z_{n, 0}, \ldots, Z_{n, m_{n}-1}\right\}_{n=1}^{\infty}$ is chosen to be $\left\{\left\lfloor n U_{n, 1}\right\rfloor, \ldots,\left\lfloor n U_{n, m_{n}}\right\rfloor\right\}_{n=1}^{\infty}$, where $\lfloor x\rfloor:=\max \{l \in \mathbb{Z}: l \leqslant x\}$ is the usual integer part of $x \in \mathbb{R}$ and $U_{n, 1}, \ldots, U_{n, m_{n}}$ for each $n$ are independent random variables uniformly distributed in the interval $(0,1)$ such that the row vectors $\left(U_{n, 1}, \ldots, U_{n, m_{n}}\right), n \in \mathbb{N}$, are also independent. In other words, the whole array $\left\{U_{n, 1}, \ldots, U_{n, m_{n}}\right\}_{n=1}^{\infty}$ consists of independent Uniform $(0,1)$ random variables, so that the model here is that each time $n$ the statistician obtains an additional observation, he will bootstrap the upgraded sample of size $n+1$ by generating $m_{n+1}$ random numbers anew, independently of all previous generations; the corresponding bootstrap means will be denoted by

$$
\mu_{n}^{\Delta}\left(m_{n}\right):=m_{n}^{-1} \sum_{j=1}^{m_{n}} X_{\left\lfloor n U_{n, j}\right\rfloor}, \quad n \in \mathbb{N} .
$$

The second model is induced by a single sequence $U_{1}, U_{2}, \ldots$ of independent Uniform $(0,1)$ random variables such that the array $\left\{Z_{n, 0}, \ldots, Z_{n, m_{n}-1}\right\}_{n=1}^{\infty}$ is $\left\{\left\lfloor n U_{1}\right\rfloor, \ldots,\left\lfloor n U_{m_{n}}\right\rfloor\right\}_{n=1}^{\infty}$. Here the rows are far from being independent: the statistician stores $U_{1}, \ldots, U_{m_{n}}$ and generates only the new random numbers $U_{m_{n}+1}, \ldots, U_{m_{n+1}}$ when the $(n+1)$ st observation comes in, and the bootstrap sample changes from $X_{\left\lfloor n U_{1}\right\rfloor}, \ldots, X_{\left\lfloor n U_{\left.m_{n}\right\rfloor}\right\rfloor}$ to $X_{\left\lfloor(n+1) U_{1}\right\rfloor}, \ldots, X_{\left\lfloor(n+1) U_{m_{n+1}}\right\rfloor}$; the corresponding bootstrap means will be denoted by

$$
\mu_{n}^{\star}\left(m_{n}\right):=m_{n}^{-1} \sum_{j=1}^{m_{n}} X_{\left\lfloor n U_{j}\right\rfloor}, \quad n \in \mathbb{N} .
$$


Clearly,

$$
\mu_{n}\left(m_{n}\right)=m_{n}^{-1} \sum_{k \in \mathscr{Z}_{n}\left(m_{n}\right)} X_{k},
$$

where the random collection $\mathscr{Z}_{n}\left(m_{n}\right):=\left\{Z_{n, 0}, \ldots, Z_{n, m_{n}-1}\right\}$ is a multiset of not necessarily different nonnegative random integers. Our method is based on analysing the joint structure of these multisets as $n \rightarrow \infty$, for the two models above and $m_{n} \equiv\left\lfloor n^{1 / \alpha}\right\rfloor$ for some $\alpha \geqslant 1$, by considering the random graphs $g_{n}\left(m_{n}\right)$ generated by $\mathscr{Z}_{n}\left(m_{n}\right)$ in a natural fashion: take $m_{n}$ (distinct) vertices representing the not necessarily different $m_{n}$ elements of $\mathscr{Z}_{n}\left(m_{n}\right)$, and connect any two vertices by an edge if the numbers they represent are the same. The problem then is about the sparsity of the edges connecting the different subgraphs $g_{n}\left(m_{n}\right)$ in the graph generated by $\cup_{n=1}^{\infty} \mathscr{Z}_{n}\left(m_{n}\right)$, for the two basic models of the bootstrap introduced above.

We are able to analyse this problem after a rarefying transformation that pertains directly to the averages

$$
a_{n}^{\Delta}(\alpha):=n^{-1} \sum_{j=1}^{n} X_{\left.\left\lfloor n^{\alpha}\right\rfloor U_{n, j}\right\rfloor} \quad \text { and } \quad a_{n}^{\star}(\alpha):=n^{-1} \sum_{j=1}^{n} X_{\left\lfloor\left\lfloor n^{\alpha}\right\rfloor U_{j}\right\rfloor}
$$

for some $\alpha \geqslant 1$, and in general to

$$
a_{n}\left(m_{n}\right):=n^{-1} \sum_{j=0}^{n-1} X_{Z_{m_{n}, j}} .
$$

The results on the corresponding random graphs are in the next section. These are very different from those in the existing standard theory, given in [8], and are of independent interest. We shall point out their direct relevance in classical random allocation problems and believe that they may be important in some recent branches of computer science. The averages $a_{n}^{\triangle}(\alpha)$ and $a_{n}^{\star}(\alpha)$ may not be as well motivated from the statistical point of view as their bootstrap-mean counterparts

$$
\mu_{n}^{\Delta}(\alpha):=\mu_{n}^{\Delta}\left(\left\lfloor n^{1 / \alpha}\right\rfloor\right)=\sum_{j=1}^{\left\lfloor n^{1 / \alpha}\right\rfloor} X_{\left\lfloor n U_{n, j}\right\rfloor} /\left\lfloor n^{1 / \alpha}\right\rfloor
$$

and

$$
\mu_{n}^{\star}(\alpha):=\mu_{n}^{\star}\left(\left\lfloor n^{1 / \alpha}\right\rfloor\right)=\sum_{j=1}^{\left\lfloor n^{1 / \alpha}\right\rfloor} X_{\left\lfloor n U_{j}\right\rfloor} /\left\lfloor n^{1 / \alpha}\right\rfloor,
$$

but they are just as interesting from the probabilistic point of view. Based on the results for random graphs in Section 2, it is in fact easier to fully characterize the complete convergence of $a_{n}^{\Delta}(\alpha)$ to $\mu$ and, at least for $\alpha>3$, the almost sure convergence of both $a_{n}^{\Delta}(\alpha)$ and $a_{n}^{\star}(\alpha)$, than to achieve corresponding statements for the bootstrap means $\mu_{n}^{\Delta}(\alpha)$ and $\mu_{n}^{\star}(\alpha)$; the results for $a_{n}\left(m_{n}\right)$ are separated in Section 3, while the main results for bootstrap means are in Section 4.

Following Hsu and Robbins [15] and Erdős [12, 13], we say that a sequence $\left\{\xi_{n}\right\}_{n=1}^{\infty}$ of random variables converges completely to the random variable $\xi$, and write $\xi_{n} \stackrel{\mathrm{HHR}}{\longrightarrow} \xi$ if 
and only if

$$
\sum_{n=1}^{\infty} \mathbb{P}\left\{\left|\xi_{n}-\xi\right|>\varepsilon\right\}<\infty
$$

for every $\varepsilon>0$. So, $\xi_{n} \stackrel{\text { EHR }}{\longrightarrow} \xi$ implies $\xi_{n} \stackrel{\text { a.s. }}{\longrightarrow} \xi$, but the converse is generally not true; in fact, to fix ideas, the Erdős-Hsu-Robbins theorem ( $c f$. [9], pp. 132, 375) says that $\bar{\mu}_{n} \stackrel{\text { EHR }}{\longrightarrow} \mu$ if and only if $\mathbb{E}\left(X^{2}\right)<\infty$, and the extension by Baum and Katz [5] states that

$$
\sum_{n=1}^{\infty} n^{\theta-2} \mathbb{P}\left\{\left|\bar{\mu}_{n}-\mu\right|>\varepsilon\right\}<\infty
$$

holds for every $\varepsilon>0$ for some $\theta>1$ if and only if $\mathbb{E}\left(|X|^{\theta}\right)<\infty$. Now the intuitive idea is that, if for the chosen model of the bootstrap the random subgraphs above are almost surely asymptotically sparsely connected, then the members of the given sequence of bootstrap means themselves become asymptotically independent, and hence the second Borel-Cantelli lemma would suggest that the strong and complete convergence of these means are equivalent. The complete convergence of them, in turn, is a universal distributional property, independent of the bootstrap model, which may be characterized by direct probabilistic methods. We are unable to accomplish this program in its full generality: conjectures arising from the work are discussed in Section 5. In each section the proofs are deferred until after the statement and discussion of the main results in that section.

\section{The sparsity of random graphs generated by random multisets}

Given a finite or infinite multiset $\mathscr{I}$ of nonnegative integers, let $S[\mathscr{I}]$ be the base set of all the different elements in $\mathscr{I}$, that is, the set that keeps exactly one of each kind of elements in $\mathscr{I}$. For $k \in \mathscr{I}$, let $\#(k, \mathscr{I})$ denote the multiplicity of $k$, so that $\#(k, S[\mathscr{I}])=1$ for every $k \in \mathscr{I}$ and $|\mathscr{I}|:=\sum_{k \in S[\mathscr{I}]} \#(k, \mathscr{I})$ is the total number of elements in $\mathscr{I}$, including all multiplicities. Given now the multisets $\mathscr{I}_{1}$ and $\mathscr{I}_{2}$ of nonnegative integers, it is natural to extend the usual set-theoretic notions and operations the following way: $\mathscr{I}_{1} \subset \mathscr{I}_{2}$ if and only if $S\left[\mathscr{I}_{1}\right] \subset S\left[\mathscr{I}_{2}\right]$ and $\#\left(k, \mathscr{I}_{1}\right) \leqslant \#\left(k, \mathscr{I}_{2}\right)$ for every $k \in S\left[\mathscr{I}_{1}\right]$, so that $\mathscr{I}_{1}=\mathscr{I}_{2}$ if and only if $S\left[\mathscr{I}_{1}\right]=S\left[\mathscr{I}_{2}\right]$ and $\#\left(k, \mathscr{I}_{1}\right)=\#\left(k, \mathscr{I}_{2}\right)$ for every $k \in S\left[\mathscr{I}_{1}\right]$; the union $\mathscr{I}_{1} \cup \mathscr{I}_{2}$ is a multiset with base set $S\left[\mathscr{I}_{1}\right] \cup S\left[\mathscr{I}_{2}\right]$ and multiplicity $\max \left(\#\left(k, \mathscr{I}_{1}\right), \#\left(k, \mathscr{I}_{2}\right)\right)$ for each $k \in S\left[\mathscr{I}_{1}\right] \cup S\left[\mathscr{I}_{2}\right]$, while the intersection $\mathscr{I}_{1} \cap \mathscr{I}_{2}$ is a multiset with base set $S\left[\mathscr{I}_{1}\right] \cap S\left[\mathscr{I}_{2}\right]$ and multiplicity $\min \left(\#\left(k, \mathscr{I}_{1}\right), \#\left(k, \mathscr{I}_{2}\right)\right)$ for each $k \in S\left[\mathscr{I}_{1}\right] \cap S\left[\mathscr{I}_{2}\right]$; finally, if $\mathscr{I}_{1}$ and $\mathscr{I}_{2}$ are both finite, the difference $\mathscr{I}_{1} \backslash \mathscr{I}_{2}$ may be formed only if $\mathscr{I}_{2} \subset \mathscr{I}_{1}$, in which case $S\left[\mathscr{I}_{1} \backslash \mathscr{I}_{2}\right]:=\left\{k \in \mathscr{I}_{1}: \#\left(k, \mathscr{I}_{1}\right)>\#\left(k, \mathscr{I}_{2}\right)\right\}$ and the multiplicity of $k \in S\left[\mathscr{I}_{1} \backslash \mathscr{I}_{2}\right]$ in $\mathscr{I}_{1} \backslash \mathscr{I}_{2}$ is taken to be $\#\left(k, \mathscr{I}_{1}\right)-\#\left(k, \mathscr{I}_{2}\right)$.

A multiset $\mathscr{I}$ of nonnegative integers generates a graph $G(\mathscr{I})$, with $|\mathscr{I}|$ distinct vertices $v_{1}, v_{2}, \ldots$, with each vertex representing an element in $\mathscr{I}$, such that there is an edge connecting two vertices if and only if the numbers that the two vertices represent are equal. Many graphs can be generated by a multiset, but one clearly sees that these graphs are isomorphic.

Keeping the notation of the introduction throughout, let $\left\{U_{n, 1}, \ldots, U_{n, n}\right\}_{n=1}^{\infty}$ be a triangular array and $U_{1}, U_{2}, \ldots$ be a sequence of independent Uniform $(0,1)$ random variables. 
For some $\alpha \geqslant 1$ and all $n \in \mathbb{N}$, consider the random multisets

$$
\mathscr{I}_{n}^{\Delta}(\alpha):=\left\{\left\lfloor\left\lfloor n^{\alpha}\right\rfloor U_{n, 1}\right\rfloor, \ldots,\left\lfloor\left\lfloor n^{\alpha}\right\rfloor U_{n, n}\right\rfloor\right\} \quad \text { and } \quad \mathscr{I}_{n}^{\star}(\alpha):=\left\{\left\lfloor\left\lfloor n^{\alpha}\right\rfloor U_{1}\right\rfloor, \ldots,\left\lfloor\left\lfloor n^{\alpha}\right\rfloor U_{n}\right\rfloor\right\},
$$

and let $G_{n}^{\triangle}(\alpha):=G\left(\mathscr{I}_{n}^{\triangle}(\alpha)\right)$ and $G_{n}^{\star}(\alpha):=G\left(\mathscr{I}_{n}^{\star}(\alpha)\right)$ be the corresponding generated graphs. If $G_{n}(\alpha)$ is any one of them, then there is an edge between any of the $\left(\begin{array}{l}n \\ 2\end{array}\right)$ pairs of vertices $v_{i}$ and $v_{j}, i, j=1, \ldots, n, i \neq j$, with probability $\mathbb{P}\left\{\left\lfloor\left\lfloor n^{\alpha}\right\rfloor U_{i}\right\rfloor=\left\lfloor\left\lfloor n^{\alpha}\right\rfloor U_{j}\right\rfloor\right\}=1 /\left\lfloor n^{\alpha}\right\rfloor$. So, when distributional matters are investigated, even asymptotically, for the isolated random multiset $\mathscr{I}_{n}^{\Delta}(\alpha)$ or $\mathscr{I}_{n}^{\star}(\alpha)$ and the corresponding generated graph $G_{n}^{\Delta}(\alpha)$ or $G_{n}^{\star}(\alpha)$, and not the joint behaviour of the whole sequence, the two constructions of the multiset may be viewed as the same and the corresponding homogeneous random graphs may also be taken to be the same. In such cases, when no distinction between the two scenarios is needed, we shall use the single sequence $U_{1}, U_{2}, \ldots$.

It follows from a well-known result of Barbour (see, for example, Theorem 5.G in [4] for a general version) that, if $\alpha>2$, then the number of edges in $G_{n}(\alpha)$ has an approximate Poisson distribution with mean $1 /\left(2 n^{\alpha-2}\right)$. However, the infinite graphs $G_{\alpha}^{\triangle}:=$ $G\left(\cup_{n=1}^{\infty} \mathscr{I}_{n}^{\Delta}(\alpha)\right)$ and $G_{\alpha}^{\star}:=G\left(\cup_{n=1}^{\infty} \mathscr{I}_{n}^{\star}(\alpha)\right)$ are inhomogeneous in the sense that the connecting probabilities between the vertices of corresponding subgraphs are no longer the same. What we really need, in both cases, is not to have many edges between $G_{n}(\alpha)$ and $G\left(\cup_{j=n+1}^{\infty} \mathscr{I}_{j}(\alpha)\right)$, within $G_{\alpha}$. Since $\mathscr{I}_{1}^{\star}(\alpha), \mathscr{I}_{2}^{\star}(\alpha), \ldots$, are not independent, this problem is clearly more difficult for $G_{\alpha}^{\star}$ than for $G_{\alpha}^{\Delta}$. Theorems 2.1 and 2.2 provide the same result for the two cases if $\alpha>3$. They say that, for every present state $n$, the number of edges leading to the whole future $n+1, n+2, \ldots$ is bounded by the same finite number, almost surely for all $n$ large enough (the threshold depending on chance).

Theorem 2.1. If $\alpha>3$, then there is a finite constant $K=K(\alpha)>0$ such that

$$
\sum_{n=1}^{\infty} \mathbb{P}\left\{\left|\mathscr{I}_{n}^{\Delta}(\alpha) \cap\left(\cup_{j=n+1}^{\infty} \mathscr{I}_{j}^{\Delta}(\alpha)\right)\right| \geqslant K\right\}<\infty .
$$

Theorem 2.2. If $\alpha>3$, then there is a finite constant $K=K(\alpha)>0$ such that

$$
\sum_{n=1}^{\infty} \mathbb{P}\left\{\left|\mathscr{I}_{n}^{\star}(\alpha) \cap\left(\cup_{j=n+1}^{\infty} \mathscr{I}_{j}^{\star}(\alpha)\right)\right| \geqslant K\right\}<\infty .
$$

Although Theorem 2.2 is much harder to prove, even $K(\alpha)$ may be chosen as

$$
K(\alpha)= \begin{cases}\lceil(\alpha-2) /(\alpha-3)\rceil, & \text { if } 3<\alpha \leqslant 4 \\ 1, & \text { if } \alpha>4\end{cases}
$$

the same in both theorems, where $\lceil x\rceil:=\min \{l \in \mathbb{Z}: x \leqslant l\}$ for any $x \in \mathbb{R}$, so that eventually there will be no edges to the future if $\alpha>4$.

For any multiset $\mathscr{I}$ of nonnegative integers, let $R[\mathscr{I}]:=R_{1}[\mathscr{I}]:=\{k: \#(k, \mathscr{I})=1\}$ be the 'regular' subset of its elements with multiplicity 1 . The following result is about the size of the regular subsets of a sequence of random multisets more general than above, that is, about the number of isolated vertices in the graphs they generate, not connected to any other vertices. As always, $\left\{m_{n}\right\}_{n=1}^{\infty}$ is a sequence of natural numbers. 
Theorem 2.3. If $\mathscr{I}_{n}^{m_{n}}=\left\{\left\lfloor m_{n} U_{1}\right\rfloor, \ldots,\left\lfloor m_{n} U_{n}\right\rfloor\right\}$ such that $\liminf _{n \rightarrow \infty} m_{n} / n>c$ for some $c>0$, then

for every $\varepsilon>0$.

$$
\lim _{n \rightarrow \infty} \mathbb{P}\left\{\frac{\left|R\left[\mathscr{I}_{n}^{m_{n}}\right]\right|}{n}>e^{-1 / c}-\varepsilon\right\}=1
$$

For $\alpha \geqslant 1$, now consider $\mathscr{J}_{n}^{\alpha}:=\left\{\left\lfloor n U_{1}\right\rfloor, \ldots,\left\lfloor n U_{\left\lfloor n^{1 / \alpha}\right\rfloor}\right\rfloor\right\}$, so that, in particular, $\mathscr{J}_{n}^{1}=$ $\mathscr{I}_{n}^{n}=\mathscr{I}_{n}(1)=: \mathscr{I}_{n}, n \in \mathbb{N}$. The next two theorems are more precise extended versions of Theorem 2.3 in more specific settings. They are not used for handling bootstrap and other means in the present paper, but, as discussed in Section 5, they may have a role in that theory as well. The results are about the number of elements in the set $R_{k}\left[\mathscr{F}_{n}^{\alpha}\right]:=\left\{0 \leqslant i \leqslant n-1: \#\left(i, \mathscr{J}_{n}^{\alpha}\right)=k\right\}$, that is, the number of elements in $\mathscr{J}_{n}^{\alpha}$ with multiplicity $k=0,1,2, \ldots$. The symbol $\stackrel{g}{\longrightarrow}$ stands for convergence in distribution and Poisson $(\lambda)$ denotes the Poisson distribution with mean $\lambda>0$.

Theorem 2.4. For every $n \in \mathbb{N}$ and $H \subset\{0\} \cup \mathbb{N}$,

$$
\mathbb{P}\left\{\left|\sum_{k \in H}\left(\frac{\left|R_{k}\left[\mathscr{J}_{n}\right]\right|}{n}-\frac{e^{-1}}{k !}\right)\right|>\varepsilon\right\} \leqslant 2 e \sqrt{n} e^{-2 n \varepsilon^{2}}
$$

for every $\varepsilon>0$.

Notice that, if $l \in \mathbb{N}$, then in graph-theoretic language $\left|R_{l}\left[\mathscr{I}_{n}\right]\right|$ is nothing but the number of subgraphs of $G\left(\mathscr{I}_{n}\right)=G\left(\left\{\left\lfloor n U_{1}\right\rfloor, \ldots,\left\lfloor n U_{n}\right\rfloor\right\}\right)$ that are isomorphic to the complete $l$ graph $K^{l}$, as defined in [7, p. 3]. Hence the theorem is about these numbers and, in particular, $\left|R_{l}\left[\mathscr{I}_{n}\right]\right| / n \stackrel{\text { EHR }}{\longrightarrow} e^{-1} / l$ ! for every $l \in \mathbb{N}$.

Theorem 2.5. Let $\alpha>1$ and consider an integer $k \in\{0\} \cup \mathbb{N}$.

(i) If $k \in[0, \alpha /(\alpha-1))$, then for every $\varepsilon>0$ there exists an $\eta(\varepsilon)>0$ such that

$$
\mathbb{P}\left\{\left|\frac{\left|R_{k}\left[\mathscr{F}_{n}^{\alpha}\right]\right|}{n\left(\left\lfloor n^{1 / \alpha}\right\rfloor / n\right)^{k}}-\frac{e^{-\left\lfloor n^{1 / \alpha}\right\rfloor / n}}{k !}\right|>\varepsilon\right\} \leqslant 2 e\left\lfloor n^{1 / \alpha}\right\rfloor^{1 / 2} e^{-\eta(\varepsilon) n\left(\left\lfloor n^{1 / \alpha}\right\rfloor / n\right)^{k}}
$$

for every $n \in \mathbb{N}$.

(ii) If $\alpha /(\alpha-1)$ is an integer and $k=\alpha /(\alpha-1)$, then $\left|R_{k}\left[\mathscr{F}_{n}^{\alpha}\right]\right| \stackrel{\mathscr{D}}{\longrightarrow}$ Poisson $(1 / k !)$.

(iii) If $k>\alpha /(\alpha-1)$, then $\mathbb{P}\left\{\left|R_{k}\left[\mathscr{J}_{n}^{\alpha}\right]\right|>\varepsilon\right\} \leqslant n^{[\alpha(1-k)-k] / \alpha} / k$ ! for every $\varepsilon>0$, where $n^{[\alpha(1-k)-k] / \alpha} \rightarrow 0$.

If $\alpha>1$ and $k \in[0, \alpha /(\alpha-1))$, then

$$
\sum_{n=1}^{\infty}\left\lfloor n^{1 / \alpha}\right\rfloor^{1 / 2} e^{-\eta(\varepsilon) n\left(\left\lfloor n^{1 / \alpha}\right] / n\right)^{k}}<\infty
$$

and so case (i) implies that

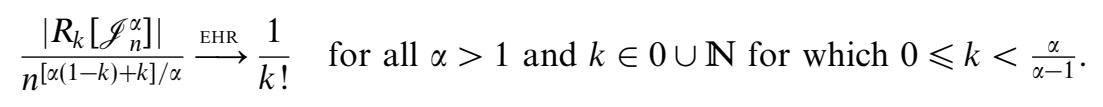


Theorems 2.4 and 2.5 may also be interpreted as results for classical random allocation problems, where the number of boxes is the original sample size $n$, while the resampling size $m=m_{n}$ is the number of balls that are equiprobably allocated into the $n$ boxes, labelled $0,1, \ldots, n-1$. Then $R_{k}(m, n):=\left|R_{k}\left[\left\{\left\lfloor n U_{1}\right\rfloor, \ldots,\left\lfloor n U_{m}\right\rfloor\right\}\right]\right|$ is the number of boxes containing $k$ balls. Markov [19] was already aware, for example, that

$$
\mathbb{P}\left\{R_{0}(m, n)=i\right\}=\frac{1}{n^{m}}\left(\begin{array}{c}
n \\
i
\end{array}\right) \sum_{j=0}^{n-i}(-1)^{n-i-j}\left(\begin{array}{c}
n-i \\
j
\end{array}\right) j^{m} .
$$

Asymptotic normal and Poisson distributions have been derived for $R_{k}(m, n)$ by many authors, under various conditions concerning the relative size of $m$ and $n$; see [18] for a detailed description of the results and some of their applications. Theorems 2.4-2.5 are different: the main point is to obtain strong laws for proportions. In principle it should be possible to derive the case $k=0$ of Theorem 2.4 from Markov's formula, for example, and the general case by more complicated methods used in [18]. However, our short proof makes more combinatorial and probabilistic sense: $R_{k}(n, n)$ is the number of complete $k$-subgraphs in the random graph $G\left(\mathscr{I}_{n}\right)$ of order $n$, the degree of each vertex of which is asymptotically Poisson with mean $n(1 / n)=1$ by the classical Poisson convergence theorem, and so the result for the proportion $R_{k}(n, n) / n$ may be viewed as the law of large numbers obtained from averaging over the individual vertices.

Proof of Theorem 2.1. Let $K=K(\alpha)$ be as given in (2.1); consider the event $A_{n}=$ $\left\{\left\lfloor\left\lfloor n^{\alpha}\right\rfloor U_{n, 1}\right\rfloor, \ldots,\left\lfloor\left\lfloor n^{\alpha}\right\rfloor U_{n, n}\right\rfloor\right.$ are different $\}=\left\{\left|R\left[\mathscr{I}_{n}^{\Delta}(\alpha)\right]\right|=n\right\}$ and the index sets $I=\{\mathbf{i}=$ $\left.\left(i_{1}, \ldots, i_{K}\right): 1 \leqslant i_{1}<\cdots<i_{K} \leqslant n\right\}, M=\left\{\mathbf{m}=\left(m_{1}, \ldots, m_{K}\right): m_{1}, \ldots, m_{K}>n\right\}$ and $J(\mathbf{m})=\left\{\mathbf{j}=\left(j_{1}, \ldots, j_{K}\right): 1 \leqslant j_{l} \leqslant m_{l}, l=1, \ldots, K\right\}$. By sequential conditioning it is easy to see that

$$
\mathbb{P}\left\{A_{n}\right\}=\prod_{k=1}^{n-1}\left(1-\left[k /\left\lfloor n^{\alpha}\right\rfloor\right]\right),
$$

whence $\mathbb{P}\left\{A_{n}^{c}\right\}=O\left(1 / n^{\alpha-2}\right)$. Also, notice that

$$
\mathbb{P}\left\{A_{n} \cap\left[\cap_{k=1}^{K}\left\{\left\lfloor\left\lfloor n^{\alpha}\right\rfloor U_{n, i_{k}}\right\rfloor=\left\lfloor\left\lfloor m_{k}^{\alpha}\right\rfloor U_{m_{k}, j_{k}}\right\rfloor\right\}\right]\right\}=0
$$

if $\left(m_{k_{1}}, j_{k_{1}}\right)=\left(m_{k_{2}}, j_{k_{2}}\right)$ for some $k_{1}, k_{2}$ such that $1 \leqslant k_{1}<k_{2} \leqslant K$, since then $\left\lfloor\left\lfloor n^{\alpha}\right\rfloor U_{n, i_{k_{1}}}\right\rfloor=$ $\left\lfloor\left\lfloor n^{\alpha}\right\rfloor U_{n, k_{2}}\right\rfloor$ necessarily. Therefore,

$$
\begin{aligned}
p_{n}^{\triangle}(\alpha) & :=\mathbb{P}\left\{A_{n} \cap\left[\cup_{\mathbf{i} \in I} \cup_{\mathbf{m} \in M} \cup_{\mathbf{j} \in J(\mathbf{m})} \cap_{k=1}^{K}\left\{\left\lfloor\left\lfloor n^{\alpha}\right\rfloor U_{n, i_{k}}\right\rfloor=\left\lfloor\left\lfloor m_{k}^{\alpha}\right\rfloor U_{m_{k}, j_{k}}\right\rfloor\right\}\right]\right\} \\
& \leqslant \sum_{\mathbf{i} \in I} \sum_{\mathbf{m} \in M} \sum_{\mathbf{j} \in J(\mathbf{m})} \mathbb{P}\left\{A_{n} \cap\left[\cap_{k=1}^{K}\left\{\left\lfloor\left\lfloor n^{\alpha}\right\rfloor U_{n, i_{k}}\right\rfloor=\left\lfloor\left\lfloor m_{k}^{\alpha}\right\rfloor U_{m_{k}, j_{k}}\right\rfloor\right\}\right]\right\} \\
& \leqslant \sum_{\mathbf{i} \in I} \sum_{\mathbf{m} \in M} \prod_{k=1}^{K} m_{k} \prod_{k=1}^{K} \frac{1}{\left\lfloor m_{k}^{\alpha}\right\rfloor} \\
& =O(1) n^{K}\left(\frac{1}{n^{\alpha-2}}\right)^{K} \\
& =\frac{O(1)}{n^{(\alpha-3) K}} .
\end{aligned}
$$


Hence

$$
\mathbb{P}\left\{\left|\mathscr{I}_{n}^{\triangle}(\alpha) \cap\left(\cup_{j=n+1}^{\infty} \mathscr{I}_{j}^{\triangle}(\alpha)\right)\right| \geqslant K\right\} \leqslant \mathbb{P}\left\{A_{n}^{c}\right\}+p_{n}^{\triangle}(\alpha)=O\left(1 / n^{\min (\alpha-2,(\alpha-3) K)}\right),
$$

proving the theorem.

Proof of Theorem 2.2. Keeping $K=K(\alpha)$ as in (2.1), consider the events

$$
\begin{aligned}
& A_{n}=\left\{\left\lfloor\left\lfloor n^{\alpha}\right\rfloor U_{1}\right\rfloor, \ldots,\left\lfloor\left\lfloor n^{\alpha}\right\rfloor U_{n}\right\rfloor \text { are different }\right\}=\left\{\left|R\left[\mathscr{I}_{n}^{\star}(\alpha)\right]\right|=n\right\}, \\
& B_{n}=\bigcup_{m>n} \bigcup_{1 \leqslant i_{1} \neq i_{2} \leqslant n} \bigcup_{1 \leqslant j_{1} \neq j_{2} \leqslant m}\left[\left\{\left\lfloor\left\lfloor m^{\alpha}\right\rfloor U_{j_{1}}\right\rfloor=\left\lfloor\left\lfloor n^{\alpha}\right\rfloor U_{i_{1}}\right\rfloor\right\} \cap\left\{\left\lfloor\left\lfloor m^{\alpha}\right\rfloor U_{j_{2}}\right\rfloor=\left\lfloor\left\lfloor n^{\alpha}\right\rfloor U_{i_{2}}\right\rfloor\right\},\right. \\
& C_{n}=\bigcup_{m_{2}>m_{1}>n} \bigcup_{1 \leqslant j \leqslant m_{1}} \bigcup_{1 \leqslant i_{1} \neq i_{2} \leqslant n}\left[\left\{\left\lfloor\left\lfloor m_{1}^{\alpha}\right\rfloor U_{j}\right\rfloor=\left\lfloor\left\lfloor n^{\alpha}\right\rfloor U_{i_{1}}\right\rfloor\right\} \cap\left\{\left\lfloor\left\lfloor m_{2}^{\alpha}\right\rfloor U_{j}\right\rfloor=\left\lfloor\left\lfloor n^{\alpha}\right\rfloor U_{i_{2}}\right\rfloor\right\},\right. \\
& D_{n}=\bigcup_{m>n} \bigcup_{1 \leqslant i \leqslant n}\left\{\left\lfloor\left\lfloor m^{\alpha}\right\rfloor U_{i}\right\rfloor=\left\lfloor\left\lfloor n^{\alpha}\right\rfloor U_{i}\right\rfloor\right\}, \\
& E_{n}=\left\{\left|\mathscr{I}_{n}^{\star}(\alpha) \cap\left(\cup_{j=n+1}^{\infty} \mathscr{I}_{j}^{\star}(\alpha)\right)\right| \geqslant K\right\},
\end{aligned}
$$

so that what we have to prove is $\sum_{n=1}^{\infty} \mathbb{P}\left\{E_{n}\right\}<\infty$. Then

$$
\mathbb{P}\left\{E_{n}\right\} \leqslant \mathbb{P}\left\{A_{n}^{c}\right\}+\mathbb{P}\left\{D_{n}\right\}+\mathbb{P}\left\{A_{n} \cap B_{n} \cap D_{n}^{c}\right\}+\mathbb{P}\left\{C_{n} \cap D_{n}^{c}\right\}+\mathbb{P}\left\{A_{n} \cap B_{n}^{c} \cap C_{n}^{c} \cap D_{n}^{c} \cap E_{n}\right\},
$$

and since we already know from the proof of Theorem 2.1 that $\mathbb{P}\left\{A_{n}^{c}\right\}=O\left(1 / n^{\alpha-2}\right)$, the theorem follows from Lemmas 2.10, 2.11 and 2.12 below.

Lemma 2.6 below is essential for Lemma 2.7, and Lemmas 2.11 and 2.12 both require Lemma 2.7, while Lemmas 2.8 and 2.9 are needed for Lemma 2.10. Throughout these seven lemmas $U_{1}, U_{2}, \ldots$ are independent $\operatorname{Uniform}(0,1)$ random variables, $U$ is a Uniform $(0,1)$ variable, $\alpha \geqslant 1$ and $K \in \mathbb{N}$ is arbitrary; so the choice $K=K(\alpha)$ in (2.1) is permissible if $\alpha>3$. The events $B_{n}, C_{n}, D_{n}$ and $E_{n}$ are those in the proof of Theorem 2.2. Lemma 2.7 requires the notion of a special kind of a bipartite graph ([7], pp. 4-5, where the notion of a connected graph and of a circuit may also be found). For some $k \in \mathbb{N}$, consider two sets $\left\{i_{1}, \ldots, i_{k}\right\}$ and $\left\{j_{1}, \ldots, j_{k}\right\}$ of positive integers, such that $i_{1}, \ldots, i_{k}$ are all different and $j_{1}, \ldots, j_{k}$ are all different, but $i_{l}=j_{m}$ is possible, $1 \leqslant l, m \leqslant k$. Let $G^{*}\left(i_{1}, \ldots, i_{k} ; j_{1}, \ldots, j_{k}\right)$ be the (bipartite) graph generated by the multiset $\left\{i_{1}, \ldots, i_{k}, j_{1}, \ldots, j_{k}\right\}$, the latter taken with the given order of its elements as listed, in which we require that, besides the existing edges between vertices that represent equal numbers, the vertices representing $i_{l}$ and $j_{l}$ are always connected, even if $i_{l} \neq j_{l}, l=1, \ldots, k$. Such a special bipartite graph, a diagram depending upon the listing order in the generating special multiset, will be called here a bigram.

Lemma 2.6. For any integers $k \in\{1, \ldots, K\}$ and $m_{j}>n \geqslant 1, j=1, \ldots, k$,

$$
p_{\alpha}\left(n, m_{1}, \ldots, m_{k}\right):=\mathbb{P}\left\{\cap_{j=1}^{k}\left\{\left\lfloor\left\lfloor n^{\alpha}\right\rfloor U_{j}\right\rfloor=\left\lfloor\left\lfloor m_{j}^{\alpha}\right\rfloor U_{j+1}\right\rfloor\right\}\right\}=O\left(\frac{1}{m_{1}^{\alpha} \cdots m_{k}^{\alpha}}\right) .
$$


Proof. For integers $l_{1}, \ldots, l_{k}$ we have

$$
p_{\alpha}\left(n, m_{1}, \ldots, m_{k}\right)=\sum_{l_{1}=0}^{\left\lfloor n^{\alpha}\right\rfloor-1} \sum_{0 \leqslant l_{2}, \ldots, l_{k} \leqslant\left\lfloor n^{\alpha}\right\rfloor-1} \mathbb{P}\left\{\cap_{j=1}^{k}\left\{\left\lfloor\left\lfloor n^{\alpha}\right\rfloor U_{j}\right\rfloor=\left\lfloor\left\lfloor m_{j}^{\alpha}\right\rfloor U_{j+1}\right\rfloor=l_{j}\right\}\right\} .
$$

For a fixed $l_{1}$, if $\left\lfloor\left\lfloor m_{1}^{\alpha}\right\rfloor U_{2}\right\rfloor=l_{1}$, then $l_{1} /\left\lfloor m_{1}^{\alpha}\right\rfloor \leqslant U_{2}<\left(l_{1}+1\right) /\left\lfloor m_{1}^{\alpha}\right\rfloor$, and so $\left\lfloor n^{\alpha}\right\rfloor U_{2} \in$ $\left[\left\lfloor n^{\alpha}\right\rfloor l_{1} /\left\lfloor m_{1}^{\alpha}\right\rfloor,\left\lfloor n^{\alpha}\right\rfloor\left(l_{1}+1\right) /\left\lfloor m_{1}^{\alpha}\right\rfloor\right)$. Since the length of this interval is $\left\lfloor n^{\alpha}\right\rfloor /\left\lfloor m_{1}^{\alpha}\right\rfloor<1$, the random integer $\left\lfloor\left\lfloor n^{\alpha}\right\rfloor U_{2}\right\rfloor$ can take at most two values, that is, for a given $l_{1}$, the number of possible values of $l_{2}=\left\lfloor\left\lfloor n^{\alpha}\right\rfloor U_{2}\right\rfloor=\left\lfloor\left\lfloor m_{2}^{\alpha}\right\rfloor U_{3}\right\rfloor$ is at most two. Consequently, continuing the argument, for any given $l_{1}$ and $j=2, \ldots, k$ the number of values of $l_{j}$ for which the probability under the sum is nonzero is at most $2^{j-1}$. Since

$$
\begin{aligned}
& \mathbb{P}\left\{\cap_{j=1}^{k}\left\{\left\lfloor\left\lfloor n^{\alpha}\right\rfloor U_{j}\right\rfloor=\left\lfloor\left\lfloor m_{j}^{\alpha}\right\rfloor U_{j+1}\right\rfloor=l_{j}\right\}\right\} \\
& \quad \leqslant \mathbb{P}\left\{\left\{\left\lfloor\left\lfloor n^{\alpha}\right\rfloor U_{1}\right\rfloor=l_{1}\right\} \cap\left[\cap_{j=1}^{k}\left\{\left\lfloor\left\lfloor m_{j}^{\alpha}\right\rfloor U_{j+1}\right\rfloor=l_{j}\right\}\right]\right\}=\frac{1}{\left\lfloor n^{\alpha}\right\rfloor\left\lfloor m_{1}^{\alpha}\right\rfloor \cdots\left\lfloor m_{k}^{\alpha}\right\rfloor}
\end{aligned}
$$

for any choice of the integers $l_{1}, \ldots, l_{k} \in\left[0,\left\lfloor n^{\alpha}\right\rfloor\right)$, this observation yields the inequality

$$
p_{\alpha}\left(n, m_{1}, \ldots, m_{k}\right) \leqslant \sum_{l_{1}=0}^{\left\lfloor n^{\alpha}\right\rfloor-1} \frac{2^{1+2+\cdots+(k-1)}}{\left\lfloor n^{\alpha}\right\rfloor\left\lfloor m_{1}^{\alpha}\right\rfloor \cdots\left\lfloor m_{k}^{\alpha}\right\rfloor}=\frac{2^{1+2+\cdots+(k-1)}}{\left\lfloor m_{1}^{\alpha}\right\rfloor \cdots\left\lfloor m_{k}^{\alpha}\right\rfloor},
$$

which (an empty sum understood as zero) is an equality for $k=1$, proving the lemma.

Lemma 2.7. Let $\left\{i_{1}, \ldots, i_{K}\right\}$ and $\left\{j_{1}, \ldots, j_{K}\right\}$ be two sets of positive integers such that $i_{l} \neq j_{l}$, $l=1, \ldots, K$. If the bigram $G^{*}=G^{*}\left(i_{1}, \ldots, i_{K} ; j_{1}, \ldots, j_{K}\right)$ has no circuit, then for arbitrary integers $m_{j}>n \geqslant 1, j=1, \ldots, K$,

$$
\mathbb{P}\left\{\cap_{l=1}^{K}\left\{\left\lfloor\left\lfloor n^{\alpha}\right\rfloor U_{i_{l}}\right\rfloor=\left\lfloor\left\lfloor m_{l}^{\alpha}\right\rfloor U_{j_{l}}\right\rfloor\right\}\right\}=O\left(\frac{1}{m_{1}^{\alpha} \cdots m_{K}^{\alpha}}\right) .
$$

Proof. Clearly, $G^{*}$ can be decomposed into $k \in\{1, \ldots, K\}$ connected bigrams $G_{r}^{*}=$ $G_{r}^{*}\left(i_{l_{1}}, \ldots, i_{l_{q(r)}} ; j_{l_{1}}, \ldots, j_{l_{q(r)}}\right)$, none of which has a circle, such that $j_{l_{s}}=i_{l_{s+1}}$ for every $s=1, \ldots, q(r)-1$ and $j_{l_{q(r)}} \neq i_{l_{1}}$, for some $q(r) \in \mathbb{N}$ for every $r=1, \ldots, k$, and there are no edges between $G_{r}^{*}$ and $G_{t}^{*}$ if $r \neq t, r, t=1, \ldots, k$. Consider

$$
F\left(G_{r}^{*}\right)=\cap_{s=1}^{q(r)}\left\{\left\lfloor\left\lfloor n^{\alpha}\right\rfloor U_{i_{l_{s}}}\right\rfloor=\left\lfloor\left\lfloor m_{l_{s}}^{\alpha}\right\rfloor U_{j_{l_{s}}}\right\rfloor\right\},
$$

an event associated with the bigram $G_{r}^{*}, r=1, \ldots, k$. Then the events $F\left(G_{1}^{*}\right), \ldots, F\left(G_{k}^{*}\right)$ are independent, owing to the lack of edges between any two of the bigrams $G_{1}^{*}, \ldots, G_{k}^{*}$, and we have

$$
\cap_{l=1}^{K}\left\{\left\lfloor\left\lfloor n^{\alpha}\right\rfloor U_{i_{l}}\right\rfloor=\left\lfloor\left\lfloor m_{l}^{\alpha}\right\rfloor U_{j_{l}}\right\rfloor\right\}=\cap_{r=1}^{k} F\left(G_{r}^{*}\right) .
$$

Applying Lemma 2.6 to each of the events $F\left(G_{r}^{*}\right), r=1, \ldots, k$, and using independence, the lemma follows.

Lemma 2.8. If $\alpha>1$, then

$$
u_{n}:=\sum_{m=n+1}^{\infty} \frac{1}{m^{\alpha}-n^{\alpha}}=O\left(\frac{\log n}{n^{\alpha-1}}\right) .
$$


Proof. We have

$$
\begin{aligned}
u_{n} & =\frac{1}{(n+1)^{\alpha}-n^{\alpha}}+\sum_{m=n+2}^{\infty} \frac{1}{m^{\alpha}-n^{\alpha}}=O(1)\left[\frac{1}{n^{\alpha-1}}+\sum_{m=n+2}^{\infty} \int_{m-1}^{m} \frac{1}{t^{\alpha}-n^{\alpha}} d t\right] \\
& =O(1)\left[\frac{1}{n^{\alpha-1}}+\frac{1}{n^{\alpha-1}} \int_{(n+1) / n}^{\infty} \frac{1}{x^{\alpha}-1} d x\right] \\
& =O(1) \frac{1}{n^{\alpha-1}}\left[1+\int_{1}^{\infty} \frac{1}{(1+y)^{\alpha}-1} d y+\int_{1 / n}^{1} \frac{1}{(1+y)^{\alpha}-1} d y\right] \\
& =O(1) \frac{1}{n^{\alpha-1}}\left[1+\int_{1 / n}^{1} \frac{1}{y} d y\right],
\end{aligned}
$$

which gives the statement.

Lemma 2.9. If $\alpha>1$, then for all integers $m>n>1$,

$$
\begin{aligned}
p_{n}^{m}(\alpha):=\mathbb{P}\left\{\left\lfloor\left\lfloor n^{\alpha}\right\rfloor U\right\rfloor=\left\lfloor\left\lfloor m^{\alpha}\right\rfloor U\right\rfloor\right\} & =\sum_{k=0}^{\left.\left\lfloor\left\lfloor n^{\alpha}\right\rfloor /\left\lfloor m^{\alpha}\right\rfloor-\left\lfloor n^{\alpha}\right\rfloor\right)\right\rfloor}\left[\frac{k+1}{\left\lfloor m^{\alpha}\right\rfloor}-\frac{k}{\left\lfloor n^{\alpha}\right\rfloor}\right] \\
& = \begin{cases}O\left(\frac{1}{m^{\alpha}-n^{\alpha}}\right), & \text { if } m \leqslant 2^{1 / \alpha} n, \\
O\left(\frac{1}{m^{\alpha}}\right), & \text { if } m>2^{1 / \alpha} n .\end{cases}
\end{aligned}
$$

Proof. We have

$$
\begin{aligned}
p_{n}^{m}(\alpha) & =\sum_{k=0}^{\left\lfloor n^{\alpha}\right\rfloor-1} \mathbb{P}\left\{\left\lfloor\left\lfloor n^{\alpha}\right\rfloor U\right\rfloor=\left\lfloor\left\lfloor m^{\alpha}\right\rfloor U\right\rfloor=k\right\} \\
& =\sum_{k=0}^{\left\lfloor n^{\alpha}\right\rfloor-1} \mathbb{P}\left\{k \leqslant\left\lfloor n^{\alpha}\right\rfloor U<k+1, k \leqslant\left\lfloor m^{\alpha}\right\rfloor U<k+1\right\} \\
& =\sum_{k=0}^{\left\lfloor n^{\alpha}\right\rfloor-1} \mathbb{P}\left\{\frac{k}{\left\lfloor n^{\alpha}\right\rfloor}<U<\frac{k+1}{\left\lfloor m^{\alpha}\right\rfloor}\right\} .
\end{aligned}
$$

Since $k /\left\lfloor n^{\alpha}\right\rfloor<(k+1) /\left\lfloor m^{\alpha}\right\rfloor$ if and only if $k<\left\lfloor n^{\alpha}\right\rfloor /\left(\left\lfloor m^{\alpha}\right\rfloor-\left\lfloor n^{\alpha}\right\rfloor\right)$, this yields the sum formula in the first statement.

As to the asymptotic behaviour of this sum, consider first the case when $m \leqslant 2^{1 / \alpha} n$. Since the terms of the sum are decreasing in $k$, we obtain

$$
p_{n}^{m}(\alpha) \leqslant\left\{1+\left\lfloor\frac{\left\lfloor n^{\alpha}\right\rfloor}{\left\lfloor m^{\alpha}\right\rfloor-\left\lfloor n^{\alpha}\right\rfloor}\right\rfloor\right\} \frac{1}{\left\lfloor m^{\alpha}\right\rfloor}=O\left(\frac{1}{m^{\alpha}-n^{\alpha}}\right) .
$$

On the other hand, if $m>2^{1 / \alpha} n$, then $\left\lfloor n^{\alpha}\right\rfloor /\left(\left\lfloor m^{\alpha}\right\rfloor-\left\lfloor n^{\alpha}\right\rfloor\right) \leqslant 1$, and hence the sum has only the terms for $k=0$ and $k=1$. Thus, in this case, $p_{n}^{m}(\alpha)<3 /\left\lfloor m^{\alpha}\right\rfloor$, completing the proof.

Lemma 2.10. If $\alpha>2$, then $\mathbb{P}\left\{D_{n}\right\}=O\left([\log n] / n^{\alpha-2}\right)$. 
Proof. By Lemma 2.9,

$$
\begin{aligned}
\mathbb{P}\left\{D_{n}\right\} & \leqslant \sum_{m=n+1}^{\infty} \sum_{i=1}^{n} \mathbb{P}\left\{\left\lfloor\left\lfloor n^{\alpha}\right\rfloor U_{i}\right\rfloor=\left\lfloor\left\lfloor m^{\alpha}\right\rfloor U_{i}\right\rfloor\right\} \\
& =O(1) \sum_{m=n+1}^{\infty} n\left[\frac{I\left(n<m \leqslant 2^{1 / \alpha} n\right)}{m^{\alpha}-n^{\alpha}}+\frac{I\left(m>2^{1 / \alpha} n\right)}{m^{\alpha}}\right] \\
& =O\left(\frac{\log n}{n^{\alpha-2}}\right),
\end{aligned}
$$

where the last step is due to Lemma 2.8

Lemma 2.11. If $\alpha>3$, then $\mathbb{P}\left\{A_{n} \cap B_{n} \cap D_{n}^{c}\right\}=O\left(1 / n^{2 \alpha-5}\right)$ and $\mathbb{P}\left\{C_{n} \cap D_{n}^{c}\right\}=O\left(1 / n^{2 \alpha-5}\right)$.

Proof. Let $\mathbb{P}\left\{\left\{\left\lfloor\left\lfloor m^{\alpha}\right\rfloor U_{j_{1}}\right\rfloor=\left\lfloor\left\lfloor n^{\alpha}\right\rfloor U_{i_{1}}\right\rfloor\right\} \cap\left\{\left\lfloor\left\lfloor m^{\alpha}\right\rfloor U_{j_{2}}\right\rfloor=\left\lfloor\left\lfloor n^{\alpha}\right\rfloor U_{i_{2}}\right\rfloor\right\} \cap A_{n} \cap D_{n}^{c}\right\}$ be denoted by $p_{i_{1}, i_{2}, j_{1}, j_{2}}^{n, m}(\alpha)$ Then, again by the subadditivity of $\mathbb{P}$,

$$
q_{n}(\alpha):=\mathbb{P}\left\{A_{n} \cap B_{n} \cap D_{n}^{c}\right\} \leqslant \sum_{m=n+1}^{\infty} \sum_{1 \leqslant i_{1} \neq i_{2} \leqslant n} \sum_{1 \leqslant j_{1} \neq j_{2} \leqslant m} p_{i_{1}, i_{2}, j_{1}, j_{2}}^{n, m}(\alpha) .
$$

If $j_{1}=i_{1}$, then $\left\{\left\lfloor\left\lfloor m^{\alpha}\right\rfloor U_{j_{1}}\right\rfloor=\left\lfloor\left\lfloor n^{\alpha}\right\rfloor U_{i_{1}}\right\rfloor\right\} \subset D_{n}$, and if $j_{2}=i_{2}$, then also $\left\{\left\lfloor\left\lfloor m^{\alpha}\right\rfloor U_{j_{2}}\right\rfloor=\right.$ $\left.\left\lfloor\left\lfloor n^{\alpha}\right\rfloor U_{i_{2}}\right\rfloor\right\} \subset D_{n}$. If $\left(j_{1}, i_{1}\right)=\left(i_{2}, j_{2}\right)$, then on the intersection of the first two events in $p_{i_{1}, i_{2}, j_{1}, j_{2}}^{n, m}(\alpha)$ we have

$$
\left\lfloor\left\lfloor n^{\alpha}\right\rfloor U_{i_{1}}\right\rfloor=\left\lfloor\left\lfloor m^{\alpha}\right\rfloor U_{j_{1}}\right\rfloor \geqslant\left\lfloor\left\lfloor n^{\alpha}\right\rfloor U_{i_{2}}\right\rfloor=\left\lfloor\left\lfloor m^{\alpha}\right\rfloor U_{j_{2}}\right\rfloor \geqslant\left\lfloor\left\lfloor n^{\alpha}\right\rfloor U_{i_{1}}\right\rfloor,
$$

implying that

$$
\left[\left\{\left\lfloor\left\lfloor m^{\alpha}\right\rfloor U_{j_{1}}\right\rfloor=\left\lfloor\left\lfloor n^{\alpha}\right\rfloor U_{i_{1}}\right\rfloor\right\} \cap\left\{\left\lfloor\left\lfloor m^{\alpha}\right\rfloor U_{j_{2}}\right\rfloor=\left\lfloor\left\lfloor n^{\alpha}\right\rfloor U_{i_{2}}\right\rfloor\right\}\right] \subset A_{n}^{c} .
$$

Hence $p_{i_{1}, i_{2}, j_{1}, j_{2}}^{n, m}(\alpha)=0$ for any one of these three cases. Thus, introducing the set

$$
H_{n}^{m}:=\left\{\left(i_{1}, i_{2}, j_{1}, j_{2}\right): 1 \leqslant i_{1} \neq i_{2} \leqslant n, 1 \leqslant j_{1} \neq j_{2} \leqslant m, j_{1} \neq i_{1}, j_{2} \neq i_{2},\left(j_{1}, i_{1}\right) \neq\left(i_{2}, j_{2}\right)\right\}
$$

and using Lemma 2.7 with $K=2$, applicable since none of the bigrams $G^{*}\left(i_{1}, i_{2} ; j_{1}, j_{2}\right)$ has a circuit if $\left(i_{1}, i_{2}, j_{1}, j_{2}\right) \in H_{n}^{m}$, we obtain

$$
\begin{aligned}
q_{n}(\alpha) & \left.\leqslant \sum_{m=n+1}^{\infty} \sum_{\left(i_{1}, i_{2}, j_{1}, j_{2}\right) \in H_{n}^{m}} \mathbb{P}\left\{\left\{\left\lfloor\left\lfloor m^{\alpha}\right\rfloor U_{j_{1}}\right\rfloor=\left\lfloor\left\lfloor n^{\alpha}\right\rfloor U_{i_{1}}\right\rfloor\right\} \cap\left\{\left\lfloor m^{\alpha}\right\rfloor U_{j_{2}}\right\rfloor=\left\lfloor\left\lfloor n^{\alpha}\right\rfloor U_{i_{2}}\right\rfloor\right\}\right\} \\
& =O(1) \sum_{m=n+1}^{\infty} \sum_{\left(i_{1}, i_{2}, j_{1}, j_{2}\right) \in H_{n}^{m}} \frac{1}{m^{2 \alpha}}=O(1) n^{2} \sum_{m=n+1}^{\infty} \frac{1}{m^{2 \alpha-2}} \\
& =O\left(\frac{1}{n^{2 \alpha-5}}\right) .
\end{aligned}
$$

Now let $\mathbb{P}\left\{\left\{\left\lfloor\left\lfloor m_{1}^{\alpha}\right\rfloor U_{j}\right\rfloor=\left\lfloor\left\lfloor n^{\alpha}\right\rfloor U_{i_{1}}\right\rfloor\right\} \cap\left\{\left\lfloor\left\lfloor m_{2}^{\alpha}\right\rfloor U_{j}\right\rfloor=\left\lfloor\left\lfloor n^{\alpha}\right\rfloor U_{i_{2}}\right\rfloor\right\} \cap D_{n}^{c}\right\}$ be denoted by $p_{n, m_{1}, m_{2}}^{j, i_{1}, i_{2}}(\alpha)$ for the proof of the second statement, consider the set

$$
M_{n}:=\left\{\left(i_{1}, i_{2}, j, m_{1}, m_{2}\right): n<m_{1}<m_{2}, 1 \leqslant i_{1} \neq i_{2} \leqslant n, i_{1} \neq j \neq i_{2}, 1 \leqslant j \leqslant m_{1}\right\},
$$


and put

$$
p_{n, m_{1}, m_{2}}^{k_{1}, k_{2}}(\alpha):=\mathbb{P}\left\{\left\lfloor\left\lfloor m_{1}^{\alpha}\right\rfloor U_{3}\right\rfloor=\left\lfloor\left\lfloor n^{\alpha}\right\rfloor U_{1}\right\rfloor=k_{1},\left\lfloor\left\lfloor m_{2}^{\alpha}\right\rfloor U_{3}\right\rfloor=\left\lfloor\left\lfloor n^{\alpha}\right\rfloor U_{2}\right\rfloor=k_{2}\right\} .
$$

Then

$$
\begin{aligned}
\mathbb{P}\left\{C_{n} \cap D_{n}^{c}\right\} & \leqslant \sum_{m_{2}>m_{1}>n} \sum_{j=1}^{m_{1}} \sum_{1 \leqslant i_{1} \neq i_{2} \leqslant n} p_{n, m_{1}, m_{2}}^{j, i_{1}, i_{2}}(\alpha) \\
& =\sum_{\left(i_{1}, i_{2}, j, m_{1}, m_{2}\right) \in M_{n}} p_{n, m_{1}, m_{2}}^{j, i_{1}, i_{2}}(\alpha) \\
& \leqslant \sum_{m_{1}=n+1}^{\infty} \sum_{m_{2}=m_{1}+1}^{\infty} m_{1} n^{2} \sum_{k_{1}, k_{2}=0}^{\left\lfloor n^{\alpha}\right\rfloor-1} p_{n, m_{1}, m_{2}}^{k_{1}, k_{2}}(\alpha) \\
& =\sum_{m_{1}=n+1}^{\infty} \sum_{m_{2}=m_{1}+1}^{\infty} \frac{m_{1} n^{2}}{\left\lfloor n^{\alpha}\right\rfloor^{2}} \sum_{k_{1}, k_{2}=0}^{\left\lfloor n^{\alpha}\right\rfloor-1} \mathbb{P}\left\{\left\lfloor\left\lfloor m_{1}^{\alpha}\right\rfloor U\right\rfloor=k_{1},\left\lfloor\left\lfloor m_{2}^{\alpha}\right\rfloor U\right\rfloor=k_{2}\right\},
\end{aligned}
$$

since $\left\{\left\lfloor\left\lfloor m_{1}^{\alpha}\right\rfloor U_{j}\right\rfloor=\left\lfloor\left\lfloor n^{\alpha}\right\rfloor U_{i_{1}}\right\rfloor\right\} \cap\left\{\left\lfloor\left\lfloor m_{2}^{\alpha}\right\rfloor U_{j}\right\rfloor=\left\lfloor\left\lfloor n^{\alpha}\right\rfloor U_{i_{2}}\right\rfloor\right\} \subset D_{n}$ if either $i_{1}=j$ or $i_{2}=j$, so nonzero terms in the first bound can result only from summing over $M_{n}$. As in the proof of Lemma 2.6, if $m_{2}>m_{1}$ and $\left\lfloor\left\lfloor m_{2}^{\alpha}\right\rfloor U\right\rfloor=k_{2}$, then $\left\lfloor\left\lfloor m_{1}^{\alpha}\right\rfloor U\right\rfloor$ can take at most two different values because, necessarily, $\left\lfloor m_{1}^{\alpha}\right\rfloor U \in\left[k_{2}\left\lfloor m_{1}^{\alpha}\right\rfloor /\left\lfloor m_{2}^{\alpha}\right\rfloor,\left(k_{2}+1\right)\left\lfloor m_{1}^{\alpha}\right\rfloor /\left\lfloor m_{2}^{\alpha}\right\rfloor\right)$ and the length of this interval is $\left\lfloor m_{1}^{\alpha}\right\rfloor /\left\lfloor m_{2}^{\alpha}\right\rfloor<1$. Therefore,

$$
\begin{aligned}
\mathbb{P}\left\{C_{n} \cap D_{n}^{c}\right\} & \leqslant 2 \sum_{m_{1}=n+1}^{\infty} \sum_{m_{2}=m_{1}+1}^{\infty} \frac{m_{1} n^{2}}{\left\lfloor n^{\alpha}\right\rfloor^{2}} \sum_{k_{2}=0}^{\left\lfloor n^{\alpha}\right\rfloor-1} \mathbb{P}\left\{\left\lfloor\left\lfloor m_{2}^{\alpha}\right\rfloor U\right\rfloor=k_{2}\right\} \\
& =2 \sum_{m_{1}=n+1}^{\infty} \sum_{m_{2}=m_{1}+1}^{\infty} \frac{m_{1} n^{2}}{\left\lfloor n^{\alpha}\right\rfloor^{2}} \frac{\left\lfloor n^{\alpha}\right\rfloor}{\left\lfloor m_{2}^{\alpha}\right\rfloor}=\frac{O(1)}{n^{\alpha-2}} \sum_{m_{1}=n+1}^{\infty} \frac{1}{m_{1}^{\alpha-2}} \\
& =O\left(\frac{1}{n^{2 \alpha-5}}\right),
\end{aligned}
$$

finishing the proof.

Lemma 2.12. If $\alpha>3$, then

$$
p_{n}^{\star}(\alpha):=\mathbb{P}\left\{A_{n} \cap B_{n}^{c} \cap C_{n}^{c} \cap D_{n}^{c} \cap E_{n}\right\}=O\left(1 / n^{(\alpha-3) K}\right)
$$

for any $K \in \mathbb{N}$ in the definition of $E_{n}$.

Proof. Put

$$
p_{\mathbf{i}, \mathbf{m}, \mathbf{j}}^{n, \alpha}:=\mathbb{P}\left\{A_{n} \cap B_{n}^{c} \cap C_{n}^{c} \cap D_{n}^{c} \cap\left[\cap_{k=1}^{K}\left\{\left\lfloor\left\lfloor n^{\alpha}\right\rfloor U_{i_{k}}\right\rfloor=\left\lfloor\left\lfloor m_{k}^{\alpha}\right\rfloor U_{j_{k}}\right\rfloor\right\}\right]\right\}
$$

and introduce the index sets

$$
\begin{aligned}
I & =\left\{\mathbf{i}=\left(i_{1}, \ldots, i_{K}\right): 1 \leqslant i_{1}<\cdots<i_{K} \leqslant n\right\} \\
M & =\left\{\mathbf{m}=\left(m_{1}, \ldots, m_{K}\right): m_{1}, \ldots, m_{K}>n\right\} \\
M_{*} & =\left\{\mathbf{m}=\left(m_{1}, \ldots, m_{K}\right): m_{1}, \ldots, m_{K}>n \text { and } m_{1}, \ldots, m_{K} \text { are different }\right\}
\end{aligned}
$$




$$
\begin{aligned}
& J(\mathbf{m})=\left\{\mathbf{j}=\left(j_{1}, \ldots, j_{K}\right): 1 \leqslant j_{l} \leqslant m_{l}, l=1, \ldots, K\right\}, \\
& J(\mathbf{m}, \mathbf{i})=\left\{\mathbf{j}=\left(j_{1}, \ldots, j_{K}\right): 1 \leqslant j_{l} \leqslant m_{l}, l=1, \ldots, K ; j_{1}, \ldots, j_{K}\right. \text { are different; } \\
& j_{l} \neq i_{l}, l=1, \ldots, K \text {; the bigram } G^{*}\left(i_{1}, \ldots, i_{K} ; j_{1}, \ldots, j_{K}\right) \\
& \text { has no circuit\}. }
\end{aligned}
$$

Then

$$
p_{n}^{\star}(\alpha) \leqslant \sum_{\mathbf{i} \in I} \sum_{\mathbf{m} \in M} \sum_{\mathbf{j} \in J(\mathbf{m})} p_{\mathbf{i}, \mathbf{m}, \mathbf{j}}^{n, \alpha}=\sum_{\mathbf{i} \in I} \sum_{\mathbf{m} \in M_{*}} \sum_{\mathbf{j} \in J(\mathbf{m}, \mathbf{i})} p_{\mathbf{i}, \mathbf{m}, \mathbf{j}}^{n, \alpha},
$$

because all other probabilities under the first sum are zero.

To see the latter claim, note first that, if $m_{l_{1}}=m_{l_{2}}$ for some $l_{1}, l_{2} \in\{1, \ldots, K\}, l_{1} \neq l_{2}$, then either $E_{\mathbf{i}, \mathbf{m}, \mathbf{j}}^{n, \alpha}:=\cap_{k=1}^{K}\left\{\left\lfloor\left\lfloor n^{\alpha}\right\rfloor U_{i_{k}}\right\rfloor=\left\lfloor\left\lfloor m_{k}^{\alpha}\right\rfloor U_{j_{k}}\right\rfloor\right\} \subset A_{n}^{c}$ or $E_{\mathbf{i}, \mathbf{m}, \mathbf{j}}^{n, \alpha} \subset B_{n}$, where the first subcase occurs if $j_{l_{1}}=j_{l_{2}}$, since then $\left\lfloor\left\lfloor n^{\alpha}\right\rfloor U_{i_{l_{1}}}\right\rfloor=\left\lfloor\left\lfloor n^{\alpha}\right\rfloor U_{i_{l_{2}}}\right\rfloor$, while the second subcase occurs if $j_{l_{1}} \neq j_{l_{2}}$. Second, if $j_{l}=i_{l}$ for some $l \in\{1, \ldots, K\}$, then $E_{\mathbf{i}, \mathbf{m}, \mathbf{j}}^{n, \alpha} \subset D_{n}$. Third, if $m_{l_{1}} \neq m_{l_{2}}$ and $j_{l_{1}}=j_{l_{2}}$ for some $l_{1}, l_{2} \in\{1, \ldots, K\}, l_{1} \neq l_{2}$, then $E_{\mathbf{i}, \mathbf{m}, \mathbf{j}}^{n, \alpha} \subset C_{n}$. Finally, suppose without loss of generality that the first three cases so far discussed do not occur, but there is a circuit in the bigram $G^{*}\left(i_{1}, \ldots, i_{K} ; j_{1}, \ldots, j_{K}\right)$. Then, again without loss of generality, the vertices connected by a circuit of edges must represent a circuit of equations of the form $j_{l}=i_{l+1}, l=1, \ldots, t-1$, and $j_{t}=i_{1}$, for some $t \in\{1, \ldots, K\}$, which implies

$$
\begin{aligned}
\left\lfloor\left\lfloor n^{\alpha}\right\rfloor U_{i_{1}}\right\rfloor & =\left\lfloor\left\lfloor m_{1}^{\alpha}\right\rfloor U_{j_{1}}\right\rfloor \geqslant\left\lfloor\left\lfloor n^{\alpha}\right\rfloor U_{i_{2}}\right\rfloor=\left\lfloor\left\lfloor m_{2}^{\alpha}\right\rfloor U_{j_{2}}\right\rfloor \geqslant \cdots \geqslant\left\lfloor\left\lfloor n^{\alpha}\right\rfloor U_{i_{t}}\right\rfloor=\left\lfloor\left\lfloor m_{k}^{\alpha}\right\rfloor U_{j_{t}}\right\rfloor \\
& \geqslant\left\lfloor\left\lfloor n^{\alpha}\right\rfloor U_{i_{1}}\right\rfloor
\end{aligned}
$$

and hence also that $E_{\mathbf{i}, \mathbf{m}, \mathbf{j}}^{n, \alpha} \subset A_{n}^{c}$. These considerations prove the claim.

Therefore, by a final application of Lemma 2.7, we obtain

$$
\begin{aligned}
p_{n}^{\star}(\alpha) & \leqslant \sum_{\mathbf{i} \in I} \sum_{\mathbf{m} \in M_{*}} \sum_{\mathbf{j} \in J(\mathbf{m}, \mathbf{i})} \mathbb{P}\left\{\cap_{k=1}^{K}\left\{\left\lfloor\left\lfloor n^{\alpha}\right\rfloor U_{i_{k}}\right\rfloor=\left\lfloor\left\lfloor m_{k}^{\alpha}\right\rfloor U_{j_{k}}\right\rfloor\right\}\right. \\
& =O(1) \sum_{\mathbf{i} \in I} \sum_{\mathbf{m} \in M_{*}} \sum_{\mathbf{j} \in J(\mathbf{m}, \mathbf{i})} \prod_{k=1}^{K} \frac{1}{m_{k}^{\alpha}} \\
& =O(1) n^{K} \sum_{\mathbf{m} \in M_{*}} \prod_{k=1}^{K} m_{k} \prod_{k=1}^{K} \frac{1}{m_{k}^{\alpha}} \\
& =O(1) n^{K}\left(\sum_{m=n}^{\infty} \frac{1}{m^{\alpha-1}}\right)^{K} \\
& =O\left(\frac{1}{n^{(\alpha-3) K}}\right),
\end{aligned}
$$

completing the proof.

Proof of Theorem 2.3. Let $G_{m_{n}}^{n}:=G\left(\mathscr{I}_{n}^{m_{n}}\right)$ be the random graph generated by $\mathscr{I}_{n}^{m_{n}}=$ $\left\{\left\lfloor m_{n} U_{1}\right\rfloor, \ldots,\left\lfloor m_{n} U_{n}\right\rfloor\right\}$, with the vertex $v_{i}$ representing $\left\lfloor m_{n} U_{i}\right\rfloor$ and having degree $d_{i}:=d\left(v_{i}\right)$, the number of edges connecting $v_{i}, i=1, \ldots, n$. Hence the random variables $d_{1}, \ldots, d_{n}$ are identically distributed and $\left|R\left[\mathscr{I}_{n}^{m_{n}}\right]\right|=\sum_{i=1}^{n} I\left(d_{i}=0\right)$. 
We have $p_{n}:=\mathbb{E}\left(I\left(d_{i}=0\right)\right)=\mathbb{P}\left\{d_{i}=0\right\}=\left[\left(m_{n}-1\right) / m_{n}\right]^{n-1}$ and

$$
q_{n}:=\mathbb{E}\left(I\left(d_{i}=0\right) I\left(d_{j}=0\right)\right)=\mathbb{P}\left\{d_{i}=0, d_{j}=0\right\}=\left[\left(m_{n}-2\right) / m_{n}\right]^{n-2}\left[\left(m_{n}-1\right) / m_{n}\right]
$$

if $i \neq j$. Hence, for any $\eta>0$, by the Markov inequality,

$$
\begin{aligned}
\mathbb{P}\left\{\left|\frac{\left|R\left[\mathscr{F}_{n}^{m_{n}}\right]\right|}{n}-p_{n}\right|>\eta\right\} & \leqslant \frac{1}{n^{2} \eta^{2}} \mathbb{E}\left(\left[\sum_{i=1}^{n}\left\{I\left(d_{i}=0\right)-p_{n}\right\}\right]^{2}\right) \\
& \leqslant \frac{1}{n^{2} \eta^{2}}\left[n+\sum_{i \neq j} \mathbb{E}\left(\left[I\left(d_{i}=0\right)-p_{n}\right]\left[I\left(d_{j}=0\right)-p_{n}\right]\right)\right] \\
& =\frac{1}{n^{2} \eta^{2}}\left[n+n(n-1)\left\{\mathbb{E}\left(I\left(d_{1}=0\right) I\left(d_{2}=0\right)\right)-p_{n}^{2}\right\}\right] \\
& =\frac{n+n(n-1)\left\{q_{n}-p_{n}^{2}\right\}}{n^{2} \eta^{2}} \\
& \leqslant \frac{C}{n}
\end{aligned}
$$

for some constant $C=C(\eta)>0$. Since, by the condition on $\left\{m_{n}\right\}$, for any given $\varepsilon>0$ the inequality $p_{n}>e^{-1 / c}-\varepsilon$ holds for all $n$ large enough, the theorem follows.

Proof of Theorem 2.4. Consider the counts $M_{i}=\left|\left\{1 \leqslant j \leqslant n:\left\lfloor n U_{j}\right\rfloor=i-1\right\}\right|$, $i=1, \ldots, n$, and notice that the vector $\left(M_{1}, \ldots, M_{n}\right)$ has the $\operatorname{Multinomial}(n ; 1 / n, \ldots, 1 / n)$ distribution. Hence, if $N_{1}, \ldots, N_{n}$ are independent Poisson(1) random variables, then

$$
\left(M_{1}, \ldots, M_{n}\right) \stackrel{\mathscr{D}}{=}\left(N_{1}, \ldots, N_{n} \mid N_{1}+\cdots+N_{n}=n\right),
$$

i.e., the distribution of $\left(M_{1}, \ldots, M_{n}\right)$ is the same as the conditional distribution of the vector $\left(N_{1}, \ldots, N_{n}\right)$, given $N_{1}+\cdots+N_{n}=n$. Therefore,

$$
\begin{aligned}
p_{n}(\varepsilon) & :=\mathbb{P}\left\{\left|\sum_{k \in H}\left[\frac{\left|R_{k}\left[\mathscr{I}_{n}\right]\right|}{n}-\frac{e^{-1}}{k !}\right]\right|>\varepsilon\right\} \\
& =\mathbb{P}\left\{\left|\sum_{k \in H} \frac{1}{n} \sum_{i=1}^{n}\left[I\left(M_{i}=k\right)-\frac{e^{-1}}{k !}\right]\right|>\varepsilon\right\} \\
& =\mathbb{P}\left\{\left|\sum_{k \in H} \frac{1}{n} \sum_{i=1}^{n}\left[I\left(N_{i}=k\right)-\frac{e^{-1}}{k !}\right]\right|>\varepsilon \mid N_{1}+\cdots+N_{n}=n\right\} \\
& \leqslant \frac{1}{\mathbb{P}\left\{N_{1}+\cdots+N_{n}=n\right\}} \mathbb{P}\left\{\left|\frac{1}{n} \sum_{i=1}^{n}\left[I\left(N_{i} \in H\right)-\mathbb{P}\left\{N_{i} \in H\right\}\right]\right|>\varepsilon\right\} \\
& \leqslant \frac{n !}{(n / e)^{n}} 2 e^{-2 n \varepsilon^{2}},
\end{aligned}
$$

where the last step is due to the exponential Chebyshev inequality in the theory of large deviations ( $c f$. Shiryaev [21, p. 69]), sometimes also referred to as the Chernoff bound. Routine calculation in an induction shows that $n ! \leqslant e n^{1 / 2}(n / e)^{n}$ for all $n \in \mathbb{N}$, proving the theorem. In fact, the stated inequality holds for all $n$ large enough for any $C>2(2 \pi)^{1 / 2}$ replacing $2 e$, as Stirling's formula shows. 
Proof of Theorem 2.5. We fix $\alpha>1$ and use the notation $m=\left\lfloor n^{1 / \alpha}\right\rfloor$ throughout.

(i) Considering first the case $k=0$ separately, we have

$$
\left|\frac{\left|R_{0}\left[\mathscr{J}_{n}^{\alpha}\right]\right|}{n}-e^{-m / n}\right| \leqslant\left|\frac{n-\mid R_{0}\left[\mathscr{J}_{n}^{\alpha}\right]}{n}\right|+\left|1-e^{-m / n}\right| \leqslant \frac{2 m}{n} \rightarrow 0,
$$

so that the claimed bound follows in a trivial fashion.

Now let $k>0$. Redefining the counts $M_{i}=\left|\left\{1 \leqslant j \leqslant m:\left\lfloor n U_{j}\right\rfloor=i-1\right\}\right|, i=1, \ldots, n$, the vector $\left(M_{1}, \ldots, M_{n}\right)$ is now Multinomial $(m ; 1 / n, \ldots, 1 / n)$, and if $N_{1}, \ldots, N_{n}$ are now independent Poisson $(m / n)$ random variables, so that $N_{1}+\cdots+N_{n}$ is Poisson $(m)$, we have $\left(M_{1}, \ldots, M_{n}\right) \stackrel{\mathscr{D}}{=}\left(N_{1}, \ldots, N_{n} \mid N_{1}+\cdots+N_{n}=m\right)$ as a version of (2.2). Therefore, denoting the probability of being estimated by $p_{n}^{k, \alpha}(\varepsilon)$, proceeding as in the previous proof and using the inequality $e^{|x|} \leqslant e^{x}+e^{-x}, x \in \mathbb{R}$, for every $\varepsilon>0$ and $t_{\varepsilon}>0$, we have

$$
\begin{aligned}
p_{n}^{k, \alpha}(\varepsilon) & =\mathbb{P}\left\{\left|\frac{1}{n} \sum_{i=1}^{n} I\left(M_{i}=k\right)-\frac{e^{-m / n}}{k !}\left(\frac{m}{n}\right)^{k}\right|>\varepsilon\left(\frac{m}{n}\right)^{k}\right\} \\
& =\mathbb{P}\left\{\left|\frac{1}{n} \sum_{i=1}^{n}\left[I\left(N_{i}=k\right)-\mathbb{P}\left\{N_{i}=k\right\}\right]\right|>\varepsilon\left(\frac{m}{n}\right)^{k} \mid N_{1}+\cdots+N_{n}=m\right\} \\
& \leqslant \frac{m !}{(m / e)^{m}} \inf _{0<t \leqslant t_{\varepsilon}}\left[\exp \left\{-\operatorname{tn} \varepsilon\left(\frac{m}{n}\right)^{k}-f_{n}^{k}(t)\right\}+\exp \left\{-\operatorname{tn\varepsilon }\left(\frac{m}{n}\right)^{k}+f_{n}^{k}(t)\right\}\right] \\
& \leqslant 2 e \sqrt{m} \exp \left\{\inf _{0<t \leqslant t_{\varepsilon}}\left[-\operatorname{tn\varepsilon }\left(\frac{m}{n}\right)^{k}+\max \left(f_{n}^{k}(t),-f_{n}^{k}(t)\right)\right]\right\},
\end{aligned}
$$

where

$f_{n}^{k}(t)=n \log \mathbb{E}\left(e^{t I\left(N_{1}=k\right)}\right)-\operatorname{tn} \mathbb{P}\left\{N_{1}=k\right\}=n \log \left(1+\left(e^{t}-1\right) \mathbb{P}\left\{N_{1}=k\right\}\right)-\operatorname{tn} \mathbb{P}\left\{N_{1}=k\right\}$.

Hence, choosing $t_{\varepsilon}:=\log (1+\varepsilon)$, by elementary considerations we obtain

$$
p_{n}^{k, \alpha}(\varepsilon) \leqslant 2 e \sqrt{m} \exp \left\{-\eta_{n}^{k, \alpha}(\varepsilon) n(m / n)^{k}\right\},
$$

where $\eta_{n}^{k, \alpha}(\varepsilon)=\min \left(\eta_{n,+}^{k, \alpha}(\varepsilon), \eta_{n,-}^{k, \alpha}(\varepsilon)\right)$, and where, using the inequality $\log (1+x) \leqslant x, x>0$,

$$
\begin{aligned}
\eta_{n,+}^{k, \alpha}(\varepsilon) & =\sup _{0<t \leqslant t_{\varepsilon}}\left\{t \varepsilon-\left(e^{t}-1-t\right) \frac{e^{-m / n}}{k !}\right\}>\sup _{0<t \leqslant t_{\varepsilon}}\left\{\varepsilon t-\left(e^{t}-1-t\right)\right\} \\
& \geqslant \varepsilon t_{\varepsilon}-\left[e^{t_{\varepsilon}}-1-t_{\varepsilon}\right]=(1+\varepsilon) \log (1+\varepsilon)-\varepsilon=: \eta_{+}(\varepsilon)>0,
\end{aligned}
$$

and, by the inequality $t=\log \left(1+\left[e^{t}-1\right]\right) \geqslant\left[e^{t}-1\right] /[1+\varepsilon], 0<t \leqslant t_{\varepsilon}$,

$$
\begin{aligned}
\eta_{n,-}^{k, \alpha}(\varepsilon) & =\sup _{0<t \leqslant t_{\varepsilon}}\left\{t \varepsilon-\left[t-\frac{e^{t}-1}{1+\varepsilon}\right] \frac{e^{-m / n}}{k !}\right\}>\sup _{0<t \leqslant t_{\varepsilon}}\left\{\varepsilon t-t+\frac{e^{t}-1}{1+\varepsilon}\right\} \\
& \geqslant \varepsilon t_{\varepsilon}-t_{\varepsilon}+\frac{e^{t_{\varepsilon}}-1}{1+\varepsilon}=\frac{\varepsilon}{1+\varepsilon}-(1-\varepsilon) \log (1+\varepsilon)=: \eta_{-}(\varepsilon)>0,
\end{aligned}
$$

proving the statement with $\eta(\varepsilon)=\min \left(\eta_{+}(\varepsilon), \eta_{-}(\varepsilon)\right)$.

(ii) We have $k=\alpha /(\alpha-1)>1$ since $\alpha>1$, and

$$
\frac{e^{-m / n}}{k !}\left(\frac{m}{n}\right)^{k}=\frac{1}{k !}\left[1+O\left(n^{(1-\alpha) / \alpha}\right)\right]
$$


Hence by Theorem 2.3.3 in [18],

$$
\mathbb{P}\left\{\left|R_{k}\left[\mathscr{J}_{n}^{\alpha}\right]\right|=l\right\}=\frac{1}{l !}\left(\frac{1}{k !}\right)^{l} e^{-1 / k !}[1+o(1)]
$$

for each $l \in\{0\} \cup \mathbb{N}$.

(iii) For every $\varepsilon>0$,

$$
\begin{aligned}
\mathbb{P}\left\{\left|R_{k}\left[\mathscr{J}_{n}^{\alpha}\right]\right|>\varepsilon\right\} & \leqslant \mathbb{P}\left\{\left|R_{k}\left[\mathscr{J}_{n}^{\alpha}\right]\right| \geqslant 1\right\} \leqslant \mathbb{E}\left(\left|R_{k}\left[\mathscr{J}_{n}^{\alpha}\right]\right|\right)=\mathbb{E}\left(\sum_{i=1}^{n} I\left(M_{i}=k\right)\right) \\
& =n \mathbb{P}\left\{M_{1}=k\right\}=n\left(\begin{array}{c}
m \\
k
\end{array}\right)\left(\frac{1}{n}\right)^{k}\left(1-\frac{1}{n}\right)^{m-k} \\
& \leqslant \frac{1}{k !} \frac{m^{k}}{n^{k-1}} \leqslant \frac{n^{[\alpha(1-k)-k] / \alpha}}{k !},
\end{aligned}
$$

completing the proof.

\section{Averages of randomly rarefied sequences}

Theorems 2.1 and 2.2 accommodate the proofs of the following results for averages of rarefied sequences more directly than those for the corresponding bootstrap analogues in the next section, the proof of which will be linked to the results here.

Theorem 3.1. If $\alpha>3$, then

$$
a_{n}^{\triangleright}(\alpha)=\frac{1}{n} \sum_{j=1}^{n} X_{\left\lfloor\left\lfloor n^{\alpha}\right\rfloor U_{n, j}\right\rfloor} \stackrel{\text { a.s. }}{\longrightarrow} \mu \quad \text { if and only if } \quad \mathbb{E}\left(X^{2}\right)<\infty
$$

and

$$
a_{n}^{\star}(\alpha)=\frac{1}{n} \sum_{j=1}^{n} X_{\left\lfloor\left\lfloor n^{\alpha}\right\rfloor U_{j}\right\rfloor} \stackrel{\text { a.s. }}{\longrightarrow} \mu \quad \text { if and only if } \quad \mathbb{E}\left(X^{2}\right)<\infty \text {. }
$$

The characterization of complete convergence can be achieved for very general rarefying sequences $\left\{m_{n}\right\}$ which are, roughly speaking, at least as fast as $\{n\}$. Here joint distributions for different $n \mathrm{~s}$ are irrelevant and hence the statement may be made in terms of a general triangular array $\left\{Z_{m_{n}, 0}, \ldots, Z_{m_{n}, n-1}\right\}_{n=1}^{\infty}$ of row-wise independent variables such that the sequence $\left\{X_{n}\right\}_{n=0}^{\infty}$ and the whole array are independent and $\mathbb{P}\left\{Z_{m_{n}, j}=k\right\}=1 / m_{n}$, $k=0, \ldots, m_{n}-1$, for every $j \in\{0, \ldots, n-1\}$ and $n \in \mathbb{N}$. Taking the special case $m_{n} \equiv\left\lfloor n^{\alpha}\right\rfloor$ in the next theorem for some $\alpha>3$ and comparing that case to Theorem 3.1 , it is of general theoretical interest to point out that almost sure convergence of the means in (3.1) and (3.2) implies their complete convergence.

Theorem 3.2. If $\liminf \inf _{n \rightarrow \infty} m_{n} / n>0$, then

$$
a_{n}\left(m_{n}\right)=\frac{1}{n} \sum_{j=0}^{n-1} X_{Z_{m_{n}, j}} \stackrel{\text { EHR }}{\longrightarrow} \mu \quad \text { if and only if } \quad \mathbb{E}\left(X^{2}\right)<\infty .
$$


In the proofs of most convergence theorems, here and in the next section, we assume without loss of generality that $\mu=\mathbb{E}(X)=0$, whenever the assumption is convenient.

Proof of Theorem 3.1. That $\mathbb{E}\left(X^{2}\right)<\infty$ is sufficient for both convergence statements in (3.1) and (3.2) follows from the sufficiency half of Theorem 3.2.

To prove necessity, let $\mu=0$, let $a_{n}(\alpha)$ denote either $a_{n}^{\triangle}(\alpha)$ or $a_{n}^{\star}(\alpha)$, and let $\mathscr{I}_{n}^{\alpha}:=\mathscr{I}_{n}(\alpha)$ denote the corresponding choice of either $\mathscr{I}_{n}^{\Delta}(\alpha)$ or $\mathscr{I}_{n}^{\star}(\alpha)$ of Theorems 2.1 and 2.2, with the corresponding regular sets

$$
R_{n}^{\alpha}:=R\left[\mathscr{I}_{n}^{\alpha} \backslash\left(\mathscr{I}_{n}^{\alpha} \cap\left[\cup_{j=n+1}^{\infty} \mathscr{I}_{j}^{\alpha}\right]\right)\right], \quad n \in \mathbb{N} .
$$

For convenience we assume, without loss of generality, that the underlying probability space $(\Omega, \mathscr{A}, \mathbb{P})$ is rich enough to carry the sequence $\left\{X_{z}\right\}_{z \in \mathbb{Z}}$ of independent and identically distributed random variables, an extended version of the given sequence $X_{0}, X_{1}, \ldots$. With $K=K(\alpha)$ meant as in (2.1), define the integer-valued random variables $T_{n}(j)$, $j=1, \ldots, n, n \in \mathbb{N}$, as follows: if $\left|R_{n}^{\alpha}\right| \leqslant n-K$, then $T_{n}(j):=-\left(n^{2}+j\right), j=1, \ldots, n$, $n \in \mathbb{N}$; while if $\left|R_{n}^{\alpha}\right|>n-K$, then $T_{n}(1), T_{n}(2), \ldots, T_{n}(n-K)$ are chosen to be the $n-K$ smallest distinct integers in $R_{n}^{\alpha}$, in the given order, and the remaining variables $T_{n}(n-K+1), T_{n}(n-K+2), \ldots, T_{n}(n)$ are chosen arbitrarily from the multiset $\mathscr{H}_{n}^{\alpha}:=$ $\mathscr{I}_{n}^{\alpha} \backslash\left\{T_{n}(1), T_{n}(2), \ldots, T_{n}(n-K)\right\}$. Clearly, any two elements of the whole triangular array $\left\{T_{n}(1), T_{n}(2), \ldots, T_{n}(n-K)\right\}_{n=K+1}^{\infty}$ are different. Since $a_{n}(\alpha) \stackrel{\text { a.s. }}{\longrightarrow} 0$, we have

$$
\begin{aligned}
0 & =\mathbb{P}\left\{\limsup _{n \rightarrow \infty}\left|a_{n}(\alpha)\right|>\varepsilon\right\} \\
& \geqslant \mathbb{P}\left\{\limsup _{n \rightarrow \infty}\left[\left\{\left|a_{n}(\alpha)\right|>\varepsilon\right\} \bigcap\left\{\left|R_{n}^{\alpha}\right|>n-K\right\}\right]\right\} \\
& \geqslant \mathbb{P}\left\{\limsup _{n \rightarrow \infty}\left[\left\{\frac{1}{n}\left|\sum_{j=1}^{n-K} X_{T_{n}(j)}\right|>2 \varepsilon\right\} \bigcap\left\{\frac{1}{n}\left|\sum_{j \in \mathscr{H}_{n}^{\alpha}} X_{j}\right| \leqslant \varepsilon\right\} \bigcap\left\{\left|R_{n}^{\alpha}\right|>n-K\right\}\right]\right\}
\end{aligned}
$$

for every $\varepsilon>0$. Since $\mathbb{E}(|X|)<\infty$, we also have

$$
\sum_{n=K}^{\infty} \mathbb{P}\left\{\left\{\frac{1}{n}\left|\sum_{j \in \mathscr{H}_{n}^{\alpha}} X_{j}\right|>\varepsilon\right\} \bigcap\left\{\left|R_{n}^{\alpha}\right|>n-K\right\}\right\}<\infty,
$$

for the number of terms in $\sum_{j \in \mathscr{H}_{n}^{\alpha}} X_{j}$ is $K$ if $\left|R_{n}^{\alpha}\right|>n-K$, whence

$$
\mathbb{P}\left\{\limsup _{n \rightarrow \infty}\left[\left\{\frac{1}{n}\left|\sum_{j=1}^{n-K} X_{T_{n}(j)}\right|>2 \varepsilon\right\} \bigcap\left\{\left|R_{n}^{\alpha}\right|>n-K\right\}\right]\right\}=0 .
$$

Since $\mathbb{P}\left\{A_{n}^{c}\right\}=\mathbb{P}\left\{\left|R\left[\mathscr{I}_{n}^{\alpha}\right]\right|<n\right\}=O\left(1 / n^{\alpha-2}\right)$ and so

$$
\sum_{n=1}^{\infty} \mathbb{P}\left\{\left|R_{n}^{\alpha}\right| \leqslant n-K\right\} \leqslant \sum_{n=1}^{\infty} \mathbb{P}\left\{A_{n}^{c}\right\}+\sum_{n=1}^{\infty} \mathbb{P}\left\{\left|\mathscr{I}_{n}^{\alpha} \cap\left[\cup_{j=n+1}^{\infty} \mathscr{I}_{j}^{\alpha}\right]\right| \geqslant K\right\}<\infty
$$

by Theorems 2.1 or 2.2 for (3.1) or for (3.2), respectively, this implies that

$$
\mathbb{P}\left\{\limsup _{n \rightarrow \infty} \frac{1}{n}\left|\sum_{j=1}^{n-K} X_{T_{n}(j)}\right|>2 \varepsilon\right\}=0 .
$$


Since the indices $\left\{T_{n}(1), T_{n}(2), \ldots, T_{n}(n-K)\right\}_{n=K+1}^{\infty}$ are all different and their whole set is independent of the sequence $\left\{X_{z}\right\}_{z \in \mathbb{Z}}$, the elements of the triangular array $\left\{X_{T_{n}(1)}, X_{T_{n}(2)}, \ldots, X_{T_{n}(n-K)}\right\}_{n=K+1}^{\infty}$ are independent and all are distributed as $X$. Therefore, by the second Borel-Cantelli lemma, it follows that

$$
\sum_{n=K+1}^{\infty} \mathbb{P}\left\{\frac{1}{n}\left|\sum_{j=1}^{n-K} X_{j}\right|>2 \varepsilon\right\}<\infty,
$$

which by Erdős's half of the Erdős-Hsu-Robbins theorem implies that $\mathbb{E}\left(X^{2}\right)<\infty$.

The necessity part of Theorem 3.2 requires the following lemma for the comparison of $n a_{n}\left(m_{n}\right)$ and the partial sums of the underlying sequence with mean $\mu$, the proof of which invokes the notation of Theorem 2.3. Since the distributional equations $a_{n}^{\triangle}\left(m_{n}\right) \stackrel{\mathscr{P}}{=} a_{n}\left(m_{n}\right) \stackrel{\mathscr{D}}{=} a_{n}^{\star}\left(m_{n}\right)$ hold for $a_{n}^{\Delta}\left(m_{n}\right):=n^{-1} \sum_{j=1}^{n} X_{\left\lfloor m_{n} U_{n, j}\right\rfloor}$ and $a_{n}^{\star}\left(m_{n}\right):=$ $n^{-1} \sum_{j=1}^{n} X_{\left\lfloor m_{n} U_{j}\right\rfloor}$ for each $n \in \mathbb{N}$, the lemma is for all these averages; for the sake of notational simplicity the proof uses $a_{n}^{\star}\left(m_{n}\right)$. Following this, Lemma 3.4 allows symmetrization in the sufficiency part of the complete convergence theorems.

Lemma 3.3. If $\liminf _{n \rightarrow \infty} m_{n} / n>c$ for some $c>0$, then, for all $\varepsilon>0$ and $\delta>0$,

$$
p_{n}^{\varepsilon}:=\mathbb{P}\left\{\left|a_{n}\left(m_{n}\right)-\mu\right|>\varepsilon\right\} \geqslant(1-\delta) \mathbb{P}\left\{\frac{1}{n}\left|\sum_{j=1}^{\left\lfloor n e^{-1 / c} / 3\right\rfloor}\left[X_{j}-\mu\right]\right|>3 \varepsilon\right\}
$$

for all $n$ large enough.

Proof. Note that $a_{n}^{\star}\left(m_{n}\right)=n^{-1} \sum_{j \in \mathscr{I}_{n}^{m_{n}}} X_{j}$. First we claim that

$$
a_{n}^{\diamond}\left(m_{n}\right):=\frac{1}{n} \sum_{j \in R\left[\mathscr{g}_{n}^{m_{n}}\right]} Y_{j} \stackrel{\mathbb{P}}{\longrightarrow} 0,
$$

where $Y_{j}=X_{j}-\mu, j \in\{0\} \cup \mathbb{N}$, and $\stackrel{\mathbb{P}}{\longrightarrow}$ denotes convergence in probability. Indeed, setting $Z_{k}=Y_{1}+\cdots+Y_{k}, k \in \mathbb{N}$, and $Z_{0}=0$, since $\left|R\left[\mathscr{I}_{n}^{m_{n}}\right]\right| \in\{0,1, \ldots, n\}$, we have

$$
\begin{aligned}
\mathbb{P}\left\{\left|a_{n}^{\diamond}\left(m_{n}\right)\right|>\varepsilon\right\} & =\sum_{k=0}^{n} \mathbb{P}\left\{\frac{1}{n}\left|\sum_{j \in R\left[\mathscr{J}_{n}^{m_{n}}\right]} Y_{j}\right|>\varepsilon|| R\left[\mathscr{I}_{n}^{m_{n}}\right] \mid=k\right\} \mathbb{P}\left\{\left|R\left[\mathscr{J}_{n}^{m_{n}}\right]\right|=k\right\} \\
& =\sum_{k=0}^{n} \mathbb{P}\left\{\frac{\left|Z_{k}\right|}{n}>\varepsilon\right\} \mathbb{P}\left\{\left|R\left[\mathscr{I}_{n}^{m_{n}}\right]\right|=k\right\}
\end{aligned}
$$

for all $\varepsilon>0$. Since $S_{k} / k \stackrel{\mathbb{P}}{\longrightarrow} \mu$ as $k \rightarrow \infty$, it is easy to see that for every $\eta>0$ there is an $n_{\varepsilon, \eta} \in \mathbb{N}$ such that $\max _{k=0,1, \ldots, n} \mathbb{P}\left\{n^{-1}\left|Z_{k}\right|>\varepsilon\right\} \leqslant \eta$ if $n \geqslant n_{\varepsilon, \eta}$. This gives (3.3).

The third statement of the theorem in [10] is that $\mu_{n}\left(m_{n}\right) \stackrel{\mathbb{P}}{\longrightarrow} \mu$, and, by a version of the easy proof, also $a_{n}\left(m_{n}\right) \stackrel{\mathbb{P}}{\longrightarrow} \mu$. This and (3.3) imply that

$$
\frac{1}{n} \sum_{j \in \mathscr{S}_{n}^{m_{n}} \backslash R\left[\mathscr{S}_{n}^{m_{n}}\right]} Y_{j} \stackrel{\mathbb{P}}{\longrightarrow} 0 .
$$


Using this relation and Theorem 2.3 and setting $b:=e^{-1 / c}$ and $\mathscr{K}_{n}:=\left\{k_{1}, \ldots, k_{n}\right\}$ for typographical convenience, for any $\varepsilon>0$ and $\eta>0$ we obtain

$$
\begin{aligned}
& p_{n}^{\varepsilon}=\sum_{k_{1}, \ldots, k_{n}=0}^{m_{n}-1} \mathbb{P}\left\{\frac{1}{n}\left|\sum_{j \in R\left[\mathscr{K}_{n}\right]} Y_{j}+\sum_{j \in \mathscr{K}_{n} \backslash R\left[\mathscr{K}_{n}\right]} Y_{j}\right|>\varepsilon\right\}\left(\frac{1}{m_{n}}\right)^{n} \\
& \geqslant \sum_{\mid R\left[\mathscr{K}_{n}\right] \geqslant\lfloor n b / 2]} \mathbb{P}\left\{\left\{\frac{1}{n}\left|\sum_{j \in R\left[\mathscr{K}_{n}\right]} Y_{j}\right|>2 \varepsilon\right\} \bigcap\left\{\frac{1}{n}\left|\sum_{j \in \mathscr{K}_{n} \backslash R\left[\mathscr{K}_{n}\right]} Y_{j}\right| \leqslant \varepsilon\right\}\right\}\left(\frac{1}{m_{n}}\right)^{n} \\
& \geqslant \sum_{\mid R\left[\mathscr{K}_{n}\right] \geqslant\lfloor n b / 2]} \min _{\lfloor n b / 2] \leqslant k \leqslant n} \mathbb{P}\left\{\frac{\left|Z_{k}\right|}{n}>2 \varepsilon\right\} \mathbb{P}\left\{\frac{1}{n}\left|\sum_{j \in \mathscr{K}_{n} \backslash R\left[\mathscr{K}_{n}\right]} Y_{j}\right| \leqslant \varepsilon\right\}\left(\frac{1}{m_{n}}\right)^{n} \\
& \geqslant \min _{\lfloor n b / 2] \leqslant k \leqslant n} \mathbb{P}\left\{\frac{\left|Z_{k}\right|}{n}>2 \varepsilon\right\}\left[\mathbb{P}\left\{\frac{1}{n}\left|\sum_{j \in \mathscr{S}_{n}^{m_{n}} \backslash R\left[\mathscr{S}_{n}^{m_{n}}\right]} Y_{j}\right| \leqslant \varepsilon\right\}-\mathbb{P}\left\{\frac{\left|R\left[\mathscr{I}_{n}^{m_{n}}\right]\right|}{n}<\frac{b}{2}\right\}\right] \\
& \geqslant \min _{\lfloor n b / 2\rfloor \leqslant k \leqslant n} \mathbb{P}\left\{\left\{\frac{\left|Z_{\lfloor n b / 3\rfloor}\right|}{n}>3 \varepsilon\right\} \bigcap\left\{\frac{\left|Z_{k}-Z_{\lfloor n b / 3\rfloor}\right|}{n}<\varepsilon\right\}\right\}\left(1-\frac{\eta}{2}\right) \\
& =\left(1-\frac{\eta}{2}\right) \mathbb{P}\left\{\frac{\left|Z_{\lfloor n b / 3\rfloor}\right|}{n}>3 \varepsilon\right\}_{\lfloor n b / 2\rfloor-\lfloor n b / 3\rfloor \leqslant k \leqslant n-\lfloor n b / 3\rfloor} \mathbb{P}\left\{\frac{\left|Z_{k}\right|}{n}<\varepsilon\right\} \\
& \geqslant\left(1-\frac{\eta}{2}\right)^{2} \mathbb{P}\left\{\frac{\left|Z_{\lfloor n b / 3\rfloor}\right|}{n}>3 \varepsilon\right\}
\end{aligned}
$$

for all large $n$, where the last step is again implied by the fact that $Z_{k} / k \stackrel{\mathbb{P}}{\longrightarrow} 0$ as $k \rightarrow \infty$.

Lemma 3.4. Let $\left\{X_{0}^{\prime}, X_{1}^{\prime}, X_{2}^{\prime}, \ldots\right\}$ be an independent copy of the basic underlying sequence $\left\{X_{0}, X_{1}, X_{2}, \ldots\right\}$ of independent and identically distributed random variables with mean $\mu \in$ $\mathbb{R}$. Consider the differences $\tilde{X}_{j}=X_{j}-X_{j}^{\prime}, j=0,1, \ldots$, symmetric about zero, and the corresponding rarefied means

$$
a_{n}\left(m_{n}\right)=n^{-1} \sum_{j=0}^{n-1} X_{Z_{m_{n}, j}}, \quad a_{n}^{\prime}\left(m_{n}\right)=n^{-1} \sum_{j=0}^{n-1} X_{Z_{m_{n}, j}}^{\prime}
$$

and

$$
\tilde{a}_{n}\left(m_{n}\right)=n^{-1} \sum_{j=0}^{n-1} \tilde{X}_{Z_{m_{n}, j}}=a_{n}\left(m_{n}\right)-a_{n}^{\prime}\left(m_{n}\right)
$$

and bootstrap means

$$
\mu_{n}\left(m_{n}\right)=m_{n}^{-1} \sum_{j=0}^{m_{n}-1} X_{Z_{n, j}}, \quad \mu_{n}^{\prime}\left(m_{n}\right)=m_{n}^{-1} \sum_{j=0}^{m_{n}-1} X_{Z_{n, j}}^{\prime}
$$

and

$$
\tilde{\mu}_{n}\left(m_{n}\right)=m_{n}^{-1} \sum_{j=0}^{m_{n}-1} \tilde{X}_{Z_{n, j}}=\mu_{n}\left(m_{n}\right)-\mu_{n}^{\prime}\left(m_{n}\right)
$$


along with the usual averages

$$
\bar{\mu}_{n}=n^{-1} \sum_{j=0}^{n-1} X_{j}, \quad \bar{\mu}_{n}^{\prime}=n^{-1} \sum_{j=0}^{n-1} X_{j}^{\prime} \quad \text { and } \quad \widetilde{\bar{\mu}}_{n}=n^{-1} \sum_{j=0}^{n-1} \tilde{X}_{j}=\bar{\mu}_{n}-\bar{\mu}_{n}^{\prime},
$$

and suppose that $m_{n} \rightarrow \infty$. Then $a_{n}\left(m_{n}\right) \stackrel{\text { EHR }}{\longrightarrow} \mu$ if and only if $\tilde{a}_{n}\left(m_{n}\right) \stackrel{\text { EHR }}{\longrightarrow} 0, \mu_{n}\left(m_{n}\right) \stackrel{\text { EHR }}{\longrightarrow} \mu$ if and only if $\tilde{\mu}_{n}\left(m_{n}\right) \stackrel{\text { EHR }}{\longrightarrow} 0$ and $\bar{\mu}_{n} \stackrel{\text { EHR }}{\longrightarrow} \mu$ if and only if $\widetilde{\bar{\mu}}_{n} \stackrel{\text { EHR }}{\longrightarrow} 0$. Furthermore, if $\xi_{1}$ and $\xi_{2}$ are independent random variables that are symmetric about zero, then $2 \mathbb{P}\left\{\left|\xi_{1}+\xi_{2}\right|>\varepsilon\right\} \geqslant$ $\mathbb{P}\left\{\left|\xi_{1}\right|>\varepsilon\right\}, \varepsilon>0$.

Proof. Let the triplet $\left(V_{n}, V_{n}^{\prime}, \tilde{V}_{n}\right)$ be either of the triplets $\left(a_{n}\left(m_{n}\right), a_{n}^{\prime}\left(m_{n}\right), \tilde{a}_{n}\left(m_{n}\right)\right),\left(\mu_{n}\left(m_{n}\right)\right.$, $\left.\mu_{n}^{\prime}\left(m_{n}\right), \tilde{\mu}_{n}\left(m_{n}\right)\right)$ and $\left(\bar{\mu}_{n}, \bar{\mu}_{n}^{\prime}, \widetilde{\bar{\mu}}_{n}\right)$, so that $V_{n}$ and $V_{n}^{\prime}$ are independent and $V_{n} \stackrel{\mathscr{D}}{=} V_{n}^{\prime}$ for every $n \in \mathbb{N}$, and let $v_{n}:=\inf \left\{x \in \mathbb{R}: \mathbb{P}\left\{V_{n} \leqslant x\right\} \geqslant \frac{1}{2}\right\}$ be the common median of $V_{n}$ and $V_{n}^{\prime}$. Then $V_{n} \stackrel{\mathbb{P}}{\longrightarrow} \mu$, as pointed out in the previous proof, which trivially implies that $v_{n} \rightarrow \mu$. Clearly,

$$
\begin{aligned}
\frac{1}{2} & \mathbb{P}\left\{\left|V_{n}-v_{n}\right|>\varepsilon\right\} \\
& =\frac{1}{2} \mathbb{P}\left\{V_{n}-v_{n}>\varepsilon\right\}+\frac{1}{2} \mathbb{P}\left\{V_{n}-v_{n}<-\varepsilon\right\} \\
& \leqslant \mathbb{P}\left\{V_{n}-v_{n}>\varepsilon, V_{n}^{\prime}-v_{n} \leqslant 0\right\}+\mathbb{P}\left\{V_{n}-v_{n}<-\varepsilon, V_{n}^{\prime}-v_{n} \geqslant 0\right\}
\end{aligned}
$$

and

$$
\mathbb{P}\left\{\left|V_{n}-V_{n}^{\prime}\right|>\varepsilon\right\} \leqslant \mathbb{P}\left\{\left\{\left|V_{n}-\mu\right|>\varepsilon / 2\right\} \cup\left\{\left|V_{n}^{\prime}-\mu\right|>\varepsilon / 2\right\}\right\},
$$

and $\left|v_{n}-\mu\right|<\varepsilon$ for all $n \geqslant n_{0}(\varepsilon)$, for any $\varepsilon>0$ for some $n_{0}(\varepsilon) \in \mathbb{N}$, and hence we obtain

$$
\frac{1}{2} \mathbb{P}\left\{\left|V_{n}-\mu\right|>2 \varepsilon\right\} \leqslant \mathbb{P}\left\{\left|\tilde{V}_{n}\right|>\varepsilon\right\} \leqslant 2 \mathbb{P}\left\{\left|V_{n}-\mu\right|>\frac{\varepsilon}{2}\right\}, \quad n \geqslant n_{0}(\varepsilon),
$$

implying all three versions of the first statement.

Similarly, we have

$$
p\left\{\left|\xi_{1}+\xi_{2}\right|>\varepsilon\right\} \geqslant \mathbb{P}\left\{\xi_{1}>\varepsilon, \xi_{2} \geqslant 0\right\}+\mathbb{P}\left\{\xi_{1}<-\varepsilon, \xi_{2} \leqslant 0\right\} \geqslant \frac{1}{2} \mathbb{P}\left\{\xi_{1}>\varepsilon\right\}+\frac{1}{2} \mathbb{P}\left\{\xi_{1}<-\varepsilon\right\},
$$

so the second statement also follows.

Proof of Theorem 3.2. Without loss of generality we assume that $\mu=0$ and, by Lemma 3.4 , also that $X$ is symmetric about zero.

Supposing then that $a_{n}\left(m_{n}\right) \stackrel{\text { EHR }}{\longrightarrow} 0$, we pick a constant $c>0$ so that $\liminf _{n \rightarrow \infty} m_{n} / n>c$, again put $b=e^{-1 / c}$, and see for the partial sums $S_{0}:=0$ and $S_{k}:=X_{1}+\cdots+X_{k}, k \in \mathbb{N}$, by Lemma 3.3 that

$$
\sum_{n=1}^{\infty} \mathbb{P}\left\{\left|S_{\lfloor n b / 3\rfloor}\right|>3 n \varepsilon\right\}<\infty .
$$

Then, with the subsequence $n(k):=\lceil 3 k / b\rceil$ we get

$$
\sum_{k=1}^{\infty} \mathbb{P}\left\{\left|S_{k}\right|>k\left(9 e^{1 / c}+3\right) \varepsilon\right\} \leqslant \sum_{k=1}^{\infty} \mathbb{P}\left\{\left|S_{\lfloor n(k) b / 3\rfloor}\right|>3 n(k) \varepsilon\right\}<\infty,
$$


and so $\mathbb{E}\left(X^{2}\right)<\infty$ again by the Erdős-Hsu-Robbins theorem.

To prove sufficiency, suppose $\mathbb{E}\left(X^{2}\right)<\infty$, and introduce the truncated variables $V_{n, j}:=$ $W_{n, j} I\left(\left|W_{n, j}\right| \leqslant n\right)$, with averages $\bar{\alpha}_{n}:=\frac{1}{n} \sum_{j=0}^{n-1} V_{n, j}$, where $W_{n, j}=X_{Z_{m_{n}, j}}, j=0,1, \ldots, n-1$. Then

$$
\begin{aligned}
\sum_{n=1}^{\infty} \mathbb{P}\left\{a_{n}\left(m_{n}\right) \neq \bar{\alpha}_{n}\right\} & \leqslant \sum_{n=1}^{\infty} \sum_{j=0}^{n-1} \mathbb{P}\left\{W_{n, j} \neq V_{n, j}\right\} \leqslant \sum_{n=1}^{\infty} \sum_{j=0}^{n-1} \mathbb{P}\{|X|>n\} \\
& =\sum_{n=1}^{\infty} n \mathbb{P}\{|X|>n\}<\infty
\end{aligned}
$$

Thus it suffices to prove that $\bar{\alpha}_{n} \stackrel{\text { EHR }}{\longrightarrow} 0$, which will follow by the Markov inequality if we show that $\sum_{n=1}^{\infty} \mathbb{E}\left(\bar{\alpha}_{n}^{4}\right)<\infty$. In the rest of the proof the constants in the $O(\cdot)$ depend only on $\inf _{n \geqslant 1} m_{n} / n$ and the underlying distribution of $X$. We have

$$
\begin{aligned}
\mathbb{E}\left(\bar{\alpha}_{n}^{4}\right) & =\frac{1}{n^{4}} \sum_{1 \leqslant j_{1}, j_{2}, j_{3}, j_{4} \leqslant n} \mathbb{E}\left(V_{n, j_{1}} V_{n, j_{2}} V_{n, j_{3}} V_{n, j_{4}}\right) \\
& =\frac{1}{n^{4}} \sum_{1 \leqslant j_{1}, j_{2}, j_{3}, j_{4} \leqslant n} \sum_{0 \leqslant k_{1}, k_{2}, k_{3}, k_{4} \leqslant m_{n}-1} \mathbb{E}\left(X_{n, k_{1}} X_{n, k_{2}} X_{n, k_{3}} X_{n, k_{4}}\right) p_{n, j_{1}, j_{2}, j_{3}, j_{4}, k_{4}, k_{3}, k_{4}}
\end{aligned}
$$

where

$$
p_{n, j_{1}, j_{2}, j_{3}, j_{4}}^{k_{1}, k_{2}, k_{3}, k_{4}}=\mathbb{P}\left\{Z_{m_{n}, j_{1}}=k_{1}, Z_{m_{n}, j_{2}}=k_{2}, Z_{m_{n}, j_{3}}=k_{3}, Z_{m_{n}, j_{4}}=k_{4}\right\}
$$

and $X_{n, k}=X_{k} I\left(\left|X_{k}\right| \leqslant n\right)$. Since $X_{n, 0}, X_{n, 1}, \ldots, X_{n, n_{n}-1}$ are independent and identically distributed symmetric random variables for each $n \in \mathbb{N}$, the expected value $\mathbb{E}\left(X_{n, k_{1}} X_{n, k_{2}} X_{n, k_{3}} X_{n, k_{4}}\right)$ may differ from zero only if either $k_{1}=k_{2}=k_{3}=k_{4}$, or $k_{1}=k_{2} \neq k_{3}=k_{4}$, or $k_{1}=k_{3} \neq k_{2}=k_{4}$, or $k_{1}=k_{4} \neq k_{2}=k_{3}$. In the first case,

$$
\sum_{1 \leqslant j_{1}, j_{2}, j_{3}, j_{4} \leqslant n} p_{n, j_{1}, j_{2}, j_{3}, j_{4}}^{k_{1}, k_{2}, k_{3}, k_{4}}=\sum_{l=1}^{4} \sum_{\left\{\left\{j_{1}, j_{2}, j_{3}, j_{4}\right\} \mid=l\right.} p_{n, j_{1}, j_{2}, j_{3}, j_{4}}^{k_{1}, k_{2}, k_{3}, k_{4}}=O(1) \sum_{l=1}^{4}\left(\frac{n}{m_{n}}\right)^{l}=O\left(\frac{n}{m_{n}}\right),
$$

while in the second, third and fourth cases,

$$
\sum_{1 \leqslant j_{1}, j_{2}, j_{3}, j_{4} \leqslant n} p_{n, j_{1}, j_{2}, j_{3}, j_{4}}^{k_{1}, k_{2}, k_{3}, k_{4}}=\sum_{l=1}^{4} \sum_{\left\{\left\{j_{1}, j_{2}, j_{3}, j_{4}\right\} \mid=l\right.} p_{n, j_{1}, j_{2}, j_{3}, j_{4}}^{k_{1}, k_{2}, k_{3}, k_{4}}=O(1) \sum_{l=2}^{4}\left(\frac{n}{m_{n}}\right)^{l}=O\left(\frac{n^{2}}{m_{n}^{2}}\right),
$$

whence

$$
\begin{aligned}
\mathbb{E}\left(\bar{\alpha}_{n}^{4}\right) & =\frac{O(1)}{n^{4}}\left[\sum_{k=0}^{m_{n}-1} \mathbb{E}\left(X_{n, k}^{4}\right) \frac{n}{m_{n}}+\sum_{0 \leqslant j<k \leqslant m_{n}-1} \mathbb{E}\left(X_{n, j}^{2}\right) \mathbb{E}\left(X_{n, k}^{2}\right) \frac{n^{2}}{m_{n}^{2}}\right] \\
& =\frac{O(1)}{n^{4}}\left[n \mathbb{E}\left(X^{4} I(|X| \leqslant n)\right)+n^{2} \mathbb{E}\left(X^{2}\right)\right] \\
& =O(1)\left[\frac{\mathbb{E}\left(X^{4} I(|X| \leqslant n)\right)}{n^{3}}+\frac{1}{n^{2}}\right] .
\end{aligned}
$$


Since

$$
\begin{aligned}
\sum_{n=1}^{\infty} \frac{\mathbb{E}\left(X^{4} I(|X| \leqslant n)\right)}{n^{3}} & =\sum_{n=1}^{\infty} \frac{1}{n^{3}} \sum_{j=1}^{n} \mathbb{E}\left(X^{4} I(j-1<|X| \leqslant j)\right) \\
& \leqslant \sum_{j=1}^{\infty} \sum_{n=j}^{\infty} \frac{j^{4}}{n^{3}} \mathbb{P}\{j-1<|X| \leqslant j\} \\
& \leqslant C \sum_{j=1}^{\infty} j^{2} \mathbb{P}\{j-1<|X| \leqslant j\}<\infty
\end{aligned}
$$

with a finite constant $C>0$, we indeed have $\sum_{n=1}^{\infty} \mathbb{E}\left(\bar{\alpha}_{n}^{4}\right)<\infty$.

\section{Bootstrap means}

The first result shows that the condition $\liminf _{n \rightarrow \infty}[\log n] / m_{n}=0$ for the bootstrap sample size $m_{n}$ is necessary for any bootstrap strong law of large numbers in both of our models when the trivial case of a degenerate underlying sequence is excluded. Some examples in [1] and [20] do suggest the result, but even the main ingredient of the proof, Lemma 4.5 for classical averages, seems to be new and of independent interest.

Theorem 4.1. If $m_{n}=O(\log n)$ and either $\mu_{n}^{\Delta}\left(m_{n}\right)=m_{n}^{-1} \sum_{j=1}^{m_{n}} X_{\left\lfloor n U_{n, j}\right\rfloor} \stackrel{\text { a.s. }}{\longrightarrow} \mu$ or $\mu_{n}^{\star}\left(m_{n}\right)=$ $m_{n}^{-1} \sum_{j=1}^{m_{n}} X_{\left\lfloor n U_{j}\right\rfloor} \stackrel{\text { a.s. }}{\longrightarrow} \mu$, then $X=\mu$ almost surely.

The next result is a counterpart of Theorem 3.1 for bootstrap means, but, unfortunately, only for necessity. Despite the apparent gap between this result and Theorem 4.4 below, however, Theorem 4.2 is good enough to effectively rule out some erroneous statements in the literature. It shows first of all that the second statement of the theorem in [10], claiming that $E\left(X^{2}\right)<\infty$ and $m_{n} \equiv\left\lfloor\log ^{1+\delta} n\right\rfloor$ for any $\delta>0$ are sufficient for the bootstrap strong law universally, is incorrect. Furthermore, Csörgő's [10] first statement and Hu's [16] theorem claim the convergence in (4.1) below whenever $\mathbb{E}\left(|X|^{\beta}\right)<\infty$ for $\beta \geqslant 2-\frac{1}{\alpha}$ and $\beta>\frac{2 \alpha}{1+\alpha}$, respectively, for any $\alpha \geqslant 1$. However, both claims are incorrect by Theorem 4.2 if $\alpha>3$ since in this case both $1+\frac{\alpha}{3}>2-\frac{1}{\alpha}$ and $1+\frac{\alpha}{3}>\frac{2 \alpha}{1+\alpha}$. (In Hu's [16] proof his inequalities (2.5)-(2.7) are clearly insufficient for his (2.8) and, even more seriously, the algebraic Markov inequality in his (2.3) cannot yield his (2.2) in the stated generality even for bounded variables.) The case with [10] is more interesting since the proof there is correct for all geometrically increasing subsequences $k_{n}(\gamma) \equiv\left\lfloor\gamma^{n}\right\rfloor, \gamma>1$, under the stated conditions (in particular, we have (4.1) below along all such $k_{n}(\gamma)$ in place of $n$ for any $\alpha \geqslant 1$ if $\left.\mathbb{E}\left(|X|^{(2 \alpha-1) / \alpha}\right)<\infty\right)$, but, as Theorem 4.2 shows, these conditions may be insufficient to control the fluctuations in the gaps of these subsequences.

Theorem 4.2. If $\alpha>3$ and either $\mu_{n}^{\Delta}(\alpha)=\left\lfloor n^{1 / \alpha}\right\rfloor^{-1} \sum_{j=1}^{\left\lfloor n^{1 / \alpha}\right\rfloor} X_{\left\lfloor n U_{n, j}\right\rfloor} \stackrel{\text { a.s. }}{\longrightarrow} \mu$ or $\mu_{n}^{\star}(\alpha)=$ $\left\lfloor n^{1 / \alpha}\right\rfloor^{-1} \sum_{j=1}^{\left\lfloor n^{1 / \alpha}\right\rfloor} X_{\left\lfloor n U_{j}\right\rfloor} \stackrel{\text { a.s. }}{\longrightarrow} \mu$, then $\mathbb{E}\left(|X|^{\beta}\right)<\infty$ for all $\beta \in\left[1,1+\frac{\alpha}{3}\right)$. 
In the last two theorems we allow a general array $\left\{Z_{n, 0}, \ldots, Z_{n, m_{n}-1}\right\}_{n=1}^{\infty}$ of row-wise independent variables, with $\mathbb{P}\left\{Z_{n, j}=k\right\}=1 / n, k=0, \ldots, n-1$, for every $j \in\left\{0, \ldots, m_{n}-1\right\}$ and $n \in \mathbb{N}$, either with $m_{n} \equiv\left\lfloor n^{1 / \alpha}\right\rfloor$ or with a general sequence of positive integers $m_{n} \rightarrow \infty$ as the bootstrap sample size, the sequence $\left\{X_{n}\right\}_{n=0}^{\infty}$ and the array being independent. Theorem 4.3 gives a characterization of complete convergence.

Theorem 4.3. If $\alpha \geqslant 1$, then

$$
\mu_{n}(\alpha):=\frac{1}{\left\lfloor n^{1 / \alpha}\right\rfloor} \sum_{j=0}^{\left\lfloor n^{1 / \alpha}\right\rfloor-1} X_{Z_{n, j}} \stackrel{\text { EHR }}{\longrightarrow} \mu \text { if and only if } \mathbb{E}\left(|X|^{1+\alpha}\right)<\infty .
$$

Starting from Theorem 4.1 (and avoiding 'irregular' sequences such as when $\left\{m_{n}\right\}$ is nondecreasing, $\liminf \operatorname{in}_{n \rightarrow \infty}[\log n] / m_{n}=0$ and $\lim \sup _{n \rightarrow \infty}[\log n] / m_{n}=\infty$ ), the slowest promising growth condition is when $m_{n} / \log n \rightarrow \infty$. Indeed, a special case of Theorem 2.2 by Arenal-Gutiérrez, Matrán and Cuesta-Albertos [1] states that (4.1) below holds in this case if $X=X_{0}$ is almost surely bounded and $X_{0}, X_{1}, X_{2}, \ldots$ are pairwise independent, identically distributed random variables. Theorem 4.4 provides 'universal' sufficient conditions for possibly unbounded variables. Part (ii) is a slight improvement of Theorem 2.1 in [1], cited in the introduction. Our proofs follow their outline; the source of the improvement is Lemma 4.7 below. The case $\alpha=1$ of part (ii) is the corresponding slight improvement of Theorem 1.2 in [1]. The comparison shows that the technique of Section 1 in [1] (which avoids truncation and uses the primary strong law for the original sample directly) is suboptimal in general. Just as in Theorem 2.1 of Arenal-Gutiérrez, Matrán and Cuesta-Albertos [1], Theorem 4.4 below requires only that the elements of the basic sequence of identically distributed random variables be pairwise independent. This is because, given $\left\{X_{0}, X_{1}, X_{2}, \ldots\right\}$, the elements of the bootstrap sample are conditionally independent regardless of the joint distribution of $X_{0}, X_{1}, X_{2}, \ldots$, and the finiteness of $\mu=\mathbb{E}(X)$ ensures $\bar{\mu}_{n} \stackrel{\text { a.s. }}{\longrightarrow} \mu$ already for pairwise independent $X_{0}, X_{1}, X_{2}, \ldots$ by Etemadi's [14] well-known theorem.

Theorem 4.4. Let $X_{0}, X_{1}, X_{2}, \ldots$ be pairwise independent.

(i) If $\mathbb{E}\left(\left[|X| \log ^{+}|X|\right]^{\alpha}\right)<\infty$ and $\liminf _{n \rightarrow \infty} m_{n} / n^{1 / \alpha}>0$ for some $\alpha \geqslant 1$, then

$$
\mu_{n}\left(m_{n}\right):=\frac{1}{m_{n}} \sum_{j=0}^{m_{n}-1} X_{Z_{n, j}} \stackrel{\text { a.s. }}{\longrightarrow} \mu .
$$

(ii) If $\mathbb{E}\left(|X|^{\alpha}\right)<\infty$ and $\liminf _{n \rightarrow \infty} m_{n} /\left[n^{1 / \alpha} \log n\right]>0$ for some $\alpha \geqslant 1$, then (4.1) also holds.

(iii) If $\mathbb{E}\left(e^{t X}\right)<\infty$ for all $t \in\left(-t_{*}, t_{*}\right)$ for some $t_{*}>0$ and $m_{n} / \log ^{2} n \rightarrow \infty$, then (4.1) holds again.

Consider again the partial sums $S_{0}=0$ and $S_{k}=X_{1}+\cdots+X_{k}, k \in \mathbb{N}$, of the basic sequence $\left\{X_{k}\right\}$ of independent variables with a finite mean $\mu=\mathbb{E}(X)$.

Lemma 4.5. If $m_{n}=O(\log n)$ and $\bar{\mu}_{m_{n}}=m_{n}^{-1} S_{m_{n}} \stackrel{\text { EHR }}{\longrightarrow} \mu$, then $X=\mu$ almost surely. 
Proof. Without loss of generality we assume that $\mu=0$ and, by Lemma 3.4, also that $X$ is symmetric about zero. Suppose, contrary to the statement, that $X$ is nondegenerate. Then the condition about complete convergence implies $m_{n} \rightarrow \infty$. Also, there is a constant $C>0$ such that $\underset{\mathbb{P}}{m_{n}} \leqslant\lfloor C \log n\rfloor$ for $n>3$.

Since $X_{1} / m_{n} \stackrel{\mathbb{P}}{\longrightarrow} 0$ and $S_{m_{n}} / m_{n} \stackrel{\mathbb{P}}{\longrightarrow} 0$, for every $\varepsilon>0$ there exists an $n_{0}=n_{0}(\varepsilon) \in \mathbb{N}$ such that $\mathbb{P}\left\{\left|S_{m_{n}}-X_{1}\right| / m_{n} \leqslant \varepsilon / C\right\} \geqslant 1 / 2$ for $n \geqslant n_{0}$. Thus

$$
\begin{aligned}
\infty & >\sum_{n=n_{0}}^{\infty} \mathbb{P}\left\{\frac{\left|S_{m_{n}}\right|}{m_{n}}>\frac{\varepsilon}{C}\right\} \geqslant \sum_{n=n_{0}}^{\infty} \mathbb{P}\left\{\frac{\left|X_{1}\right|}{m_{n}}>\frac{2 \varepsilon}{C}, \frac{\left|S_{m_{n}}-X_{1}\right|}{m_{n}} \leqslant \frac{\varepsilon}{C}\right\} \\
& \geqslant \frac{1}{2} \sum_{n=n_{0}}^{\infty} \mathbb{P}\{|X|>2 \varepsilon \log n\},
\end{aligned}
$$

which implies that $\phi(t):=\mathbb{E}\left(e^{t X}\right)<\infty$ for every $t \in \mathbb{R}$. Then by standard large deviation theory (see $\left[22\right.$, p. 8] for the form we use), $\left[\mathbb{P}\left\{S_{n} \geqslant n t\right\}\right]^{1 / n} \rightarrow \rho(t):=\phi\left(h_{t}\right) e^{-h_{t} t}$ for any $t>0$, where $h_{t}$ is the unique solution $h$ of the equation $t=\phi^{\prime}(h) / \phi(h)$.

Note that $\phi(t)=\phi(-t)=\mathbb{E}\left(e^{t X}+e^{-t X}\right) / 2 \geqslant 1$ for all $t \in \mathbb{R}$ because $X$ is symmetric. Put $\tau:=\min \left(\log (10 e / 27), \phi^{\prime}(1) / \phi(1)\right)$. The variable $X$ being nondegenerate, the function $\psi(t):=(\log \phi(t))^{\prime}=\phi^{\prime}(t) / \phi(t), t \geqslant 0$, is strictly increasing, which implies $0<h_{\tau} \leqslant 1$, and hence also the inequality $\rho(\tau)=\phi\left(h_{\tau}\right) e^{-h_{\tau} \tau} \geqslant e^{-\tau} \geqslant \frac{2.7}{e}$. Since $\psi^{\prime}(t)>0, t \geqslant 0$, it also follows that $\rho(\eta \tau)>\rho(\tau)$ if $0<\eta<1$, and so by the large deviation theorem for every $\eta \in(0,1)$ there exists an $n_{*}=n_{*}(\eta, \tau) \in \mathbb{N}$ such that

$$
\infty=\sum_{n=n_{*}}^{\infty}\left(\frac{2.6}{e}\right)^{\log n} \leqslant \sum_{n=n_{*}}^{\infty} \rho^{m_{n}}(\tau) \leqslant \sum_{n=n_{*}}^{\infty} \mathbb{P}\left\{\frac{S_{m_{n}}}{m_{n}}>\eta \tau\right\}<\infty,
$$

where the last sum is finite in view of the assumption that $\bar{\mu}_{m_{n}} \stackrel{\text { EHR }}{\longrightarrow} 0$. This contradiction implies that $X$ is degenerate.

Proof of Theorem 4.1. Again, we may and shall assume that $\mu=0$ and, by Lemma 3.4, that $X$ is symmetric about zero. Assuming

$$
\mu_{n}^{\star}\left(m_{n}\right)=m_{n}^{-1} \sum_{j=1}^{m_{n}} X_{\left\lfloor n U_{j}\right\rfloor} \stackrel{\text { a.s. }}{\longrightarrow} 0,
$$

we first consider the single-sequence set-up and introduce the multiset $\mathscr{M}_{n}:=\mathscr{I}_{m_{n^{4}}}^{n^{4}}=$ $\left\{\left\lfloor n^{4} U_{1}\right\rfloor, \ldots,\left\lfloor n^{4} U_{m_{n^{4}}}\right\rfloor\right\}$ and the events

$$
E_{n}:=\left\{\left|\mathscr{M}_{n} \cap\left(\cup_{j=n+1}^{\infty} \mathscr{M}_{j}\right)\right| \geqslant 1\right\} \quad \text { and } \quad A_{n}:=\left\{\left|R\left[\mathscr{M}_{n}\right]\right|=m_{n^{4}}\right\},
$$

redefining the corresponding events in the proof of Theorem 2.2. Then

$$
\begin{aligned}
\mathbb{P}\left\{E_{n}\right\} & \leqslant \mathbb{E}\left(\left|\mathscr{M}_{n} \cap\left(\cup_{j=n+1}^{\infty} \mathscr{M}_{j}\right)\right|\right) \leqslant \sum_{i=1}^{m_{n^{4}}} \sum_{k=n+1}^{\infty} \sum_{j=1}^{m_{k^{4}}} \mathbb{P}\left\{\left\lfloor n^{4} U_{i}\right\rfloor=\left\lfloor k^{4} U_{j}\right\rfloor\right\} \\
& \leqslant \sum_{i=1}^{m_{n^{4}}} \sum_{k=n+1}^{\infty} O(1)\left[\frac{m_{k^{4}}}{k^{4}}+\frac{I\left(k \leqslant 2^{1 / 4} n\right)}{k^{4}-n^{4}}+\frac{I\left(k>2^{1 / 4} n\right)}{k^{4}}\right]=O(1) \frac{\log n}{n^{2}}
\end{aligned}
$$


by applications of Lemmas 2.9 and 2.8, and

$$
\mathbb{P}\left\{A_{n}\right\}=\prod_{j=1}^{m_{n^{4}}-1}\left(1-\frac{j}{n^{4}}\right)=1+O(1) \frac{m_{n^{4}}^{2}}{n^{4}}=1+O\left(\frac{\log ^{2} n}{n^{4}}\right),
$$

whence $\sum_{n=1}^{\infty} \mathbb{P}\left\{A_{n}^{c} \cup E_{n}\right\}<\infty$.

Introducing now the integer-valued random variables

$$
\tau_{n}(j)(\omega):=\left\{\begin{array}{ll}
\left\lfloor n^{4} U_{j}(\omega)\right\rfloor, & \text { if } \omega \in A_{n} \cap E_{n}^{c}, \\
-\left(n^{2}+j\right), & \text { if } \omega \notin A_{n} \cap E_{n}^{c},
\end{array} \quad j=1,2, \ldots m_{n^{4}} ; n \in \mathbb{N},\right.
$$

and extending without loss of generality the sequence $\left\{X_{k}\right\}_{k=0}^{\infty}$ to the sequence $\left\{X_{z}\right\}_{z \in \mathbb{Z}}$ of independent and identically distributed random variables, for every $\varepsilon>0$ we obtain

$$
\begin{aligned}
0 & =\lim _{n \rightarrow \infty} \mathbb{P}\left\{\bigcup_{k=n}^{\infty}\left\{\frac{1}{m_{k^{4}}}\left|\sum_{j=1}^{m_{k^{4}}} X_{\left\lfloor k^{4} U_{j}\right\rfloor}\right|>\varepsilon\right\}\right\} \\
& \geqslant \limsup _{n \rightarrow \infty} \mathbb{P}\left\{\bigcup_{k=n}^{\infty}\left[\left\{\frac{1}{m_{k^{4}}}\left|\sum_{j=1}^{m_{k^{4}}} X_{\tau_{k}(j)}\right|>\varepsilon\right\} \cap A_{k} \cap E_{k}^{c}\right]\right\} \\
& \geqslant \limsup _{n \rightarrow \infty}\left[\mathbb{P}\left\{\bigcup_{k=n}^{\infty}\left\{\frac{1}{m_{k^{4}}}\left|\sum_{j=1}^{m_{k^{4}}} X_{\tau_{k}(j)}\right|>\varepsilon\right\}\right\}-\sum_{k=n}^{\infty} \mathbb{P}\left\{A_{k}^{c} \cup E_{k}\right\}\right] \\
& =\limsup _{n \rightarrow \infty} \mathbb{P}\left\{\bigcup_{k=n}^{\infty}\left\{\frac{1}{m_{k^{4}}}\left|\sum_{j=1}^{m_{k^{4}}} X_{\tau_{k}(j)}\right|>\varepsilon\right\}\right\} .
\end{aligned}
$$

Since $\tau_{n}(j) \neq \tau_{n^{\prime}}\left(j^{\prime}\right)$ if $(n, j) \neq\left(n^{\prime}, j^{\prime}\right)$, the array $\left\{X_{\tau_{n}(1)}, \ldots, X_{\tau_{n}\left(m_{n^{4}}\right)}\right\}_{n=1}^{\infty}$ consists of independent random variables, all of which are distributed as $X$, and hence by the second Borel-Cantelli lemma

$$
\frac{1}{m_{n^{4}}} \sum_{j=1}^{m_{n^{4}}} X_{j} \stackrel{\text { EHR }}{\longrightarrow} 0 .
$$

Noting that $m_{n^{4}}=O(\log n)$, the sequence version of the theorem now follows from Lemma 4.5 .

The triangular array version has exactly the same proof: replacing $U_{1}, \ldots, U_{m_{n^{4}}}$ by $U_{m_{n^{4}}, 1}, \ldots, U_{m_{n^{4}}, m_{n^{4}}}$ throughout, the only change is that the inequality in (4.2) simplifies to

$$
\mathbb{P}\left\{E_{n}\right\} \leqslant \sum_{i=1}^{m_{n}{ }^{4}} \sum_{k=n+1}^{\infty} O(1) \frac{1}{k^{4}}=O\left((\log n) / n^{3}\right) .
$$

Lemma 4.6. If $\alpha>3$ and $\mathbb{E}\left(|X|^{p}\right)<\infty$ for some $p \in[1, \alpha / 3)$, then either

$$
\mu_{n}^{\triangleright}(\alpha)=\sum_{j=1}^{\left\lfloor n^{1 / \alpha}\right\rfloor} X_{\left\lfloor n U_{n, j}\right\rfloor} /\left\lfloor n^{1 / \alpha}\right\rfloor \stackrel{\text { a.s. }}{\longrightarrow} \mu \quad \text { or } \quad \mu_{n}^{\star}(\alpha)=\sum_{j=1}^{\left\lfloor n^{1 / \alpha}\right\rfloor} X_{\left\lfloor n U_{j}\right\rfloor} /\left\lfloor n^{1 / \alpha}\right\rfloor \stackrel{\text { a.s. }}{\longrightarrow} \mu
$$

implies that $\mathbb{E}\left(|X|^{p+1}\right)<\infty$. 
Proof. Take $\mu=0$ again. First assuming $\mu_{n}^{\star}(\alpha) \stackrel{\text { a.s. }}{\longrightarrow} 0$, consider the subsequences $n_{l}:=$ $\left\lfloor l^{\alpha / p}\right\rfloor$ and $r_{l}:=\left\lfloor n_{l}^{1 / \alpha}\right\rfloor$, the multisets $\mathscr{I}_{l}^{*}:=\mathscr{I}_{r_{l}}^{n_{l}}=\left\{n_{l} U_{1}, \ldots, n_{l} U_{r_{l}}\right\}$ and corresponding regular sets $R_{l}^{*}:=R\left[\mathscr{I}_{l}^{*} \backslash\left(\mathscr{I}_{l}^{*} \cap\left[\cup_{j=l+1}^{\infty} \mathscr{I}_{j}^{*}\right]\right)\right], l \in \mathbb{N}$. Since $r_{l} \leqslant l$ and $\alpha / p>3$, by Theorem 2.2 we have

$$
\sum_{l=1}^{\infty} \mathbb{P}\left\{\left|\mathscr{I}_{l}^{*} \cap\left(\cup_{j=l+1}^{\infty} \mathscr{I}_{j}^{*}\right)\right| \geqslant K\right\}<\infty
$$

for some $K=K(\alpha / p) \in \mathbb{N}$. Furthermore,

$$
\mathbb{P}\left\{\left|R\left[\mathscr{I}_{l}^{*}\right]\right|<r_{l}\right\}=1-\prod_{k=1}^{r_{l}-1}\left(1-\frac{k}{n_{l}}\right)=O\left(1 / l^{(\alpha-2) / p}\right)
$$

as $l \rightarrow \infty$, where $(\alpha-2) / p>1$ because $\alpha-2>\alpha / 3>p$ for $\alpha>3$. Also, having $\mathbb{E}\left(|X|^{p}\right)<\infty$ and the number of terms in $\sum_{j \in \mathscr{I}_{l}^{*} \backslash R_{l}^{*}} X_{j}$ less than $K$ if $\left|R_{l}^{*}\right|>l-K$, it follows that

$$
\sum_{l=K}^{\infty} \mathbb{P}\left\{\left\{\frac{1}{r_{l}}\left|\sum_{j \in \mathscr{I}_{l}^{*} \backslash R_{l}^{*}} X_{j}\right|>\varepsilon\right\} \bigcap\left\{\left|R_{l}^{*}\right|>l-K\right\}\right\}<\infty .
$$

Thus, since finally $\mu_{r_{l}}^{\star}(\alpha) \stackrel{\text { a.s. }}{\longrightarrow} 0$ as $l \rightarrow \infty$, we have the four basic ingredients to allow a version of the proof of Theorem 3.1, which yields

$$
\frac{1}{r_{l}} \sum_{j=1}^{r_{l}-K} X_{j} \stackrel{\text { EHR }}{\longrightarrow} 0, \quad \text { and hence } \quad \bar{\mu}_{\left\lfloor l^{1 / p}\right\rfloor}=\frac{1}{\left\lfloor l^{1 / p}\right\rfloor} \sum_{j=1}^{\left\lfloor l^{1 / p}\right\rfloor} X_{j} \stackrel{\text { EHR }}{\longrightarrow} 0 \quad \text { as } \quad l \rightarrow \infty,
$$

where, in the last implication, we again use that $\mathbb{E}\left(|X|^{p}\right)<\infty$. Spelling this out,

$$
\infty>\sum_{l=1}^{\infty} \mathbb{P}\left\{\bar{\mu}_{\left\lfloor l^{1 / p}\right\rfloor}>\varepsilon\right\}=\sum_{n=1}^{\infty} \sum_{l=\left\lceil n^{p}\right\rceil}^{\left\lceil(n+1)^{p}\right\rceil-1} \mathbb{P}\left\{\bar{\mu}_{n}>\varepsilon\right\} \geqslant C_{p} \sum_{n=1}^{\infty} n^{p-1} \mathbb{P}\left\{\bar{\mu}_{n}>\varepsilon\right\}
$$

for every $\varepsilon>0$ and a finite constant $C_{p}>0$. By the theorem of Baum and Katz [5] cited at the end of the introduction, this implies that $\mathbb{E}\left(|X|^{p+1}\right)<\infty$.

The proof for $\mu_{n}^{\triangle}(\alpha)$ is the same, with reference to Theorem 2.1 instead of Theorem 2.2.

Proof of Theorem 4.2. Let $\mu_{n}$ stand for either $\mu_{n}^{\triangle}(\alpha)$ or $\mu_{n}^{\star}(\alpha)$, and consider the subsequence $n(l):=\left\lfloor l^{\alpha}\right\rfloor, l \in \mathbb{N}$, for the given $\alpha>3$. Then $\mu_{n(l)} \stackrel{\text { a.s. }}{\longrightarrow} \mu$ as $l \rightarrow \infty$, and so $\mathbb{E}\left(X^{2}\right)<\infty$ by an application of Theorem 3.1 .

Now for each $\beta \in\left(2,1+\frac{\alpha}{3}\right)$ define $H_{\beta}:=\left\{k \in \mathbb{N}: k \leqslant\lfloor\beta\rfloor\right.$ and $\left.\mathbb{E}\left(|X|^{k+\{\beta\}}\right)<\infty\right\}$, where $\{\beta\}:=\beta-\lfloor\beta\rfloor$ is the fractional part of $\beta$. Notice that $\mathbb{E}\left(|X|^{1+\{\beta\}}\right)<\infty$ since $\mathbb{E}\left(X^{2}\right)<\infty$, so $H_{\beta} \neq \emptyset$. We claim that $k_{\beta}:=\max \left\{k: k \in H_{\beta}\right\}=\lfloor\beta\rfloor$. Indeed, if $k_{\beta}<\lfloor\beta\rfloor$, then $k_{\beta}+\{\beta\} \leqslant\lfloor\beta\rfloor+\{\beta\}-1=\beta-1<\frac{\alpha}{3}$, and since $\mu_{n} \stackrel{\text { a.s. }}{\longrightarrow} \mu$, we have $\mathbb{E}\left(|X|^{k_{\beta}+1+\{\beta\}}\right)<\infty$ by Lemma 4.6, and hence $k_{\beta}+1 \in H_{\beta}$, contradicting the definition of $k_{\beta}$. Therefore, $\mathbb{E}\left(|X|^{\beta}\right)=\mathbb{E}\left(|X|^{\mid \beta\rfloor+\{\beta\}}\right)<\infty$ for any $\beta \in[1,1+\alpha / 3)$.

Proof of Theorem 4.3. Assuming $\mu_{n}(\alpha) \stackrel{\text { EHR }}{\longrightarrow} \mu$, first consider necessity. Introduce the sequence $\left\{m_{k}\right\}_{k=1}^{\infty}$ defined by $m_{k}=n$ if $\left\lfloor(n-1)^{1 / \alpha}\right\rfloor<k \leqslant\left\lfloor n^{1 / \alpha}\right\rfloor, n \in \mathbb{N}$. Then $m_{k} / k \rightarrow \infty$ 
as $k \rightarrow \infty$ and $m_{\left\lfloor n^{1 / \alpha}\right\rfloor}=n$ for all $n \in \mathbb{N}$. Thus $\mu_{n}(\alpha)=a_{\left\lfloor n^{1 / \alpha\rfloor}\right\rfloor}(n)=a_{\left\lfloor n^{1 / \alpha}\right\rfloor}\left(m_{\left\lfloor n^{1 / \alpha}\right\rfloor}\right)$, in the notation of Theorem 3.2, and so, using the assumption and Lemma 3.3, we see that, for every $b=e^{-1 / c} \in(0,1)$ and $\varepsilon>0$,

$$
\begin{aligned}
\infty & >\sum_{n=1}^{\infty} \mathbb{P}\left\{\frac{1}{\left\lfloor n^{1 / \alpha}\right\rfloor}\left|\sum_{j=1}^{\left\lfloor\left\lfloor 1^{1 / \alpha} b / 3\right\rfloor\right.}\left[X_{j}-\mu\right]\right|>\varepsilon\right\} \\
& \geqslant \sum_{k=1}^{\infty} \sum_{n=\left[\lceil 3 k / b]^{\alpha}\right\rceil}^{\left[\lfloor(k+1) / b]^{\alpha}\right]-1} \mathbb{P}\left\{\frac{1}{k}\left|\sum_{j=1}^{k}\left[X_{j}-\mu\right]\right|>\frac{6 \varepsilon}{b}\right\} \geqslant C_{\alpha}^{b} \sum_{k=1}^{\infty} k^{\alpha-1} \mathbb{P}\left\{\left|\bar{\mu}_{n}-\mu\right|>\frac{6 \varepsilon}{b}\right\}
\end{aligned}
$$

for some constant $C_{\alpha}^{b}>0$. Thus $\mathbb{E}\left(|X|^{1+\alpha}\right)<\infty$, again by the Baum-Katz theorem.

To prove sufficiency, suppose $\mathbb{E}\left(|X|^{1+\alpha}\right)<\infty$ and, without loss of generality by Lemma 3.4, also that $X$ is symmetric about $\mu=0$. Since the case $\alpha=1$ is covered by the special case $m_{n} \equiv n$ in Theorem 3.2, suppose that $\alpha>1$ and define $k_{\alpha}:=\lceil(2+2 \alpha) /(\alpha-1)\rceil$. Using the notation of Theorem 2.5, let $G\left(\mathscr{J}_{n}^{\alpha}\right)$ be the random graph generated by the multiset $\mathscr{J}_{n}^{\alpha}=\left\{\left\lfloor n U_{1}\right\rfloor, \ldots,\left\lfloor n U_{m_{n}}\right\rfloor\right\}$, with the vertex $v_{j}$ of degree $d_{j}=d\left(v_{j}\right)$ representing $n U_{j}$, $j=1, \ldots, m_{n}$, where $m_{n}=\left\lfloor n^{1 / \alpha}\right\rfloor$. As in the proof of Theorem 2.3, the degrees $d_{1}, \ldots, d_{m_{n}}$ are identically distributed, but now

$$
\mathbb{P}\left\{d_{1}=l\right\}=\left(\begin{array}{c}
m_{n}-1 \\
l
\end{array}\right)\left(\frac{1}{n}\right)^{l}\left(1-\frac{1}{n}\right)^{m_{n}-1-l} \quad l=0,1, \ldots, m_{n}-1 .
$$

For all $n$ large enough, these probabilities decrease in $l=k_{\alpha}, \ldots, m_{n}-1$, and so

$$
\mathbb{P}\left\{d_{1} \geqslant k_{\alpha}\right\} \leqslant m_{n} \mathbb{P}\left\{d_{1}=k_{\alpha}\right\} \leqslant m_{n}\left(\begin{array}{c}
m_{n}-1 \\
k_{\alpha}
\end{array}\right)\left(\frac{1}{n}\right)^{k_{\alpha}} \leqslant \frac{1}{n^{(2 \alpha+1) / \alpha}} .
$$

Hence, setting $p_{n}^{\alpha}(\varepsilon):=\mathbb{P}\left\{\left|\mu_{n}(\alpha)\right|>\varepsilon\right\}$ and using also the last statement of Lemma 3.4,

$$
\begin{aligned}
p_{n}^{\alpha}(\varepsilon) & \leqslant \mathbb{P}\left\{\left\{\left|\mu_{n}(\alpha)\right|>\varepsilon\right\} \cap\left[\cap_{j=1}^{m_{n}}\left\{d_{j}<k_{\alpha}\right\}\right]\right\}+\mathbb{P}\left\{\cup_{j=1}^{m_{n}}\left\{d_{j} \geqslant k_{\alpha}\right\}\right\} \\
& \leqslant \mathbb{P}\left\{\frac{1}{m_{n}}\left|\sum_{k=1}^{k_{\alpha}} \sum_{j \in R_{k}\left[\mathscr{F}_{n}^{\alpha}\right]} k X_{j}\right|>\varepsilon\right\}+m_{n} \mathbb{P}\left\{d_{1} \geqslant k_{\alpha}\right\} \\
& \leqslant \sum_{k=1}^{k_{\alpha}} \mathbb{P}\left\{\frac{1}{m_{n}}\left|\sum_{j \in R_{k}\left[\mathscr{F}_{n}^{\alpha}\right]} X_{j}\right|>\frac{\varepsilon}{k_{\alpha}^{2}}\right\}+\frac{m_{n}}{n^{(2 \alpha+1) / \alpha}} \\
& \leqslant \sum_{k=1}^{k_{\alpha}} \sum_{l=1}^{m_{n}} \mathbb{P}\left\{\frac{1}{m_{n}}\left|\sum_{j \in R_{k}\left[\mathscr{F}_{n}^{\alpha}\right]} X_{j}\right|>\frac{\varepsilon}{k_{\alpha}^{2}}|| R_{k}\left[\mathscr{F}_{n}^{\alpha}\right] \mid=l\right\} \mathbb{P}\left\{\left|R_{k}\left[\mathscr{J}_{n}^{\alpha}\right]\right|=l\right\}+\frac{1}{n^{2}} \\
& =\sum_{k=1}^{k_{\alpha}} \sum_{l=1}^{m_{n}} \mathbb{P}\left\{\frac{1}{m_{n}}\left|S_{l}\right|>\frac{\varepsilon}{k_{\alpha}^{2}}\right\} \mathbb{P}\left\{\left|R_{k}\left[\mathscr{J}_{n}^{\alpha}\right]\right|=l\right\}+\frac{1}{n^{2}} \\
& \leqslant 2 \sum_{k=1}^{k_{\alpha}} \sum_{l=1}^{m_{n}} \mathbb{P}\left\{\frac{1}{m_{n}}\left|S_{l}+\left(S_{m_{n}}-S_{l}\right)\right|>\frac{\varepsilon}{k_{\alpha}^{2}}\right\} \mathbb{P}\left\{\left|R_{k}\left[\mathscr{J}_{n}^{\alpha}\right]\right|=l\right\}+\frac{1}{n^{2}} \\
& \leqslant 2 k_{\alpha} \mathbb{P}\left\{\frac{\left|S_{m_{n}}\right|}{m_{n}}>\frac{\varepsilon}{k_{\alpha}^{2}}\right\}+\frac{1}{n^{2}}
\end{aligned}
$$


for every $\varepsilon>0$, where $S_{j}=X_{1}+\cdots+X_{j}, j \in \mathbb{N}$. Since, for some constant $K_{\alpha}>0$,

$$
\sum_{n=1}^{\infty} \mathbb{P}\left\{\frac{\left|S_{m_{n}}\right|}{m_{n}}>\frac{\varepsilon}{k_{\alpha}^{2}}\right\}=\sum_{j=1}^{\infty} \sum_{n=\left\lceil j^{\alpha}\right\rceil}^{\left\lceil(j+1)^{\alpha}\right\rceil-1} \mathbb{P}\left\{\frac{\left|S_{j}\right|}{j}>\frac{\varepsilon}{k_{\alpha}^{2}}\right\} \leqslant K_{\alpha} \sum_{j=1}^{\infty} j^{\alpha-1} \mathbb{P}\left\{\frac{\left|S_{j}\right|}{j}>\frac{\varepsilon}{k_{\alpha}^{2}}\right\}<\infty
$$

on account of the finiteness of $\mathbb{E}\left(|X|^{1+\alpha}\right)$, as an application of the sufficiency half of the Baum-Katz theorem, it follows that $\sum_{n=1}^{\infty} \mathbb{P}\left\{\left|\mu_{n}(\alpha)\right|>\varepsilon\right\}<\infty$ for all $\varepsilon>0$.

Lemma 4.7. If $Y$ is a nonnegative random variable with $\mathbb{E}(Y)<\infty$, then there exists a function $r:[0, \infty) \mapsto[1, \infty)$ such that $r(\cdot)$ is nondecreasing and $x / r(x), 0 \leqslant x<\infty$, is increasing, $\lim _{x \rightarrow \infty} r(x)=\infty, \lim _{x \rightarrow \infty} x / r(x)=\infty$ and $\int_{0}^{\infty} \mathbb{P}\{Y>x / r(x)\} d x<\infty$.

Proof. Since $\mathbb{E}(Y)<\infty$, we have $\int_{0}^{\infty} \mathbb{P}\{Y>x\} d x<\infty$, so for any $k \in \mathbb{N}$ there exists a $u_{k}>0$ such that $\int_{u_{k}}^{\infty} \mathbb{P}\{Y>x\} d x<2^{-k}$. Clearly, $\left\{u_{k}\right\}_{k=1}^{\infty}$ can be taken to be strictly increasing. Putting $u_{0}:=0$ and defining the continuous function

$$
g(t):= \begin{cases}k+1, & \text { if } t \in\left[u_{2 k}, u_{2 k+1}\right], k=0,1,2, \ldots, \\ k+1+\frac{t-u_{2 k+1}}{u_{2 k+2}-u_{2 k+1}}, & \text { if } t \in\left[u_{2 k+1}, u_{2 k+2}\right], k=0,1,2, \ldots,\end{cases}
$$

we have

$$
\begin{aligned}
\int_{0}^{\infty} \mathbb{P}\{Y>t\} g(t) d t & =\sum_{k=0}^{\infty} \int_{u_{2 k}}^{u_{2 k+2}} \mathbb{P}\{Y>t\} g(t) d t \leqslant \sum_{k=0}^{\infty}(k+2) \int_{u_{2 k}}^{u_{2 k+2}} \mathbb{P}\{Y>t\} d t \\
& \leqslant 2 u_{2}+\sum_{k=1}^{\infty} \frac{k+2}{2^{2 k}}<\infty .
\end{aligned}
$$

Now let $G(x):=\int_{0}^{x} g(t) d t, 0 \leqslant x<\infty$, a monotone increasing, continuously differentiable function with $G(\infty)=\infty$. Hence its inverse function $G^{-1}(\cdot)$, a one-to-one mapping of $[0, \infty)$ onto $[0, \infty)$, also has these properties; note that $G^{-1}(x)=x, 0 \leqslant x \leqslant u_{1}$. Thus, defining $r(x):=x / G^{-1}(x)$, the function $x / r(x), 0 \leqslant x<\infty$, is increasing to $\infty$. Furthermore, $r(x)=1$ if $0 \leqslant x \leqslant u_{1}$, and $r(x) \uparrow \infty$ as $x \rightarrow \infty$ if and only if $r(G(t))=G(t) / t \uparrow \infty$ as $t \rightarrow \infty$, which obviously is the case by L'Hôpital's rule; so $r(\cdot)$ in fact strictly increases to $\infty$ on $\left[u_{1}, \infty\right)$. Finally, we have $\int_{0}^{\infty} \mathbb{P}\{Y>x / r(x)\} d x=\int_{0}^{\infty} \mathbb{P}\{Y>t\} g(t) d t<\infty$ by construction.

Proof of Theorem 4.4. (i) By Lemma 4.7 there exists a positive sequence $\left\{r_{n}\right\}_{n \in \mathbb{N}}$ such that $r_{n} \uparrow \infty, n / r_{n} \uparrow \infty$ and

$$
S(\alpha):=\sum_{n=1}^{\infty} \mathbb{P}\left\{\left(|X| \log ^{+}|X|\right)^{\alpha}>\frac{n}{r_{n}}\right\}<\infty .
$$

For each $n \in \mathbb{N}$, let $t_{n}>1$ be the unique solution $x$ of the equation $\left(x \log ^{+} x\right)^{\alpha}=$ $(x \log x)^{\alpha}=n / r_{n}, x>1$. Setting $t_{0}:=t_{1}$, the sequence $\left\{t_{n}\right\}_{n=0}^{\infty}$ is necessarily nondecreasing, $t_{n} \uparrow \infty$ and

$$
\sum_{n=1}^{\infty} \mathbb{P}\left\{|X|>t_{n}\right\} \leqslant S(\alpha)<\infty
$$


For the truncated variables $X_{n}^{*}:=X_{n} I\left(\left|X_{n}\right| \leqslant t_{n}\right), n \in\{0\} \cup \mathbb{N}$, the latter implies that $\sum_{n=0}^{\infty} \mathbb{P}\left\{X_{n}^{*} \neq X_{n}\right\}<\infty$ and hence, in particular, also that $n^{-1} \sum_{j=0}^{n-1} X_{j}^{*} \stackrel{\text { a.s. }}{\longrightarrow} \mu$. Now, returning to the notation at the beginning of the introduction, let $Y_{n, 0}^{*}, \ldots, Y_{n, m_{n}-1}^{*}$ denote the variables in the bootstrap sample corresponding to the truncated sample $X_{0}^{*}, \ldots, X_{n-1}^{*}$, and for $\varepsilon>0$ introduce the conditional probabilities

$$
P_{n}(\epsilon):=\mathbb{P}\left\{\left|\frac{1}{m_{n}} \sum_{j=0}^{m_{n}-1} Y_{n, j}^{*}-\frac{1}{n} \sum_{j=0}^{n-1} X_{j}^{*}\right|>\varepsilon \mid \mathbf{X}\right\}=: \mathbb{Q} \mathbf{X}\left\{\left|\sum_{j=0}^{m_{n}-1} Y_{n, j}^{*}-\frac{m_{n}}{n} \sum_{j=0}^{n-1} X_{j}^{*}\right|>m_{n} \varepsilon\right\},
$$

given the whole sequence $\mathbf{X}=\left\{X_{n}\right\}_{n=0}^{\infty}$. If we show that $\sum_{n=1}^{\infty} P_{n}(\varepsilon)<\infty$ almost surely for each $\varepsilon>0$, then $\mathbb{P}\{\mathbb{P}\{A \mid \mathbf{X}\}=1\}=1$ and so $\mathbb{P}\{A\}=1$, by a conditional application of the Borel-Cantelli lemma, where

$$
A=\left\{m_{n}^{-1} \sum_{j=0}^{m_{n}-1} Y_{n, j}^{*}-n^{-1} \sum_{j=0}^{n-1} X_{j}^{*} \rightarrow 0\right\} .
$$

Hence

$$
m_{n}^{-1} \sum_{j=0}^{m_{n}-1} Y_{n, j}^{*}=\sum_{k=0}^{n-1} w_{n, k} X_{k}^{*} \stackrel{\text { a.s. }}{\longrightarrow} \mu,
$$

which by Lemma 2.1 in [1] implies that

$$
\mu_{n}\left(m_{n}\right)=m_{n}^{-1} \sum_{j=0}^{m_{n}-1} Y_{n, j}=\sum_{k=0}^{n-1} w_{n, k} X_{k} \stackrel{\text { a.s. }}{\longrightarrow} \mu .
$$

Since under $\mathbb{Q}_{\mathbf{X}}(\cdot)=\mathbb{P}\{\cdot \mid \mathbf{X}\}$ the variables $Y_{n, 0}^{*}, \ldots, Y_{n, m_{n}-1}^{*}$ are independent, for which we almost surely have $\left|Y_{n, j}^{*}\right| \leqslant t_{n}, \mathbb{E}_{\mathbb{Q}_{\mathbf{X}}}\left(Y_{n, j}^{*}\right)=n^{-1} \sum_{i=0}^{n-1} X_{i}^{*}$ and

$$
\operatorname{Var}_{\mathbb{Q}_{\mathbf{X}}}\left(Y_{n, j}^{*}\right) \leqslant \mathbb{E}_{\mathbb{Q}_{\mathbf{X}}}\left(\left[Y_{n, j}^{*}\right]^{2}\right) \leqslant t_{n} n^{-1} \sum_{i=0}^{n-1}\left|X_{i}^{*}\right| \leqslant t_{n} V, \quad j=0,1, \ldots, m_{n}-1,
$$

where $V$ is a proper (almost surely finite) positive random variable not depending on $n$, copying the proof of Theorem 2.1 in [1] and so applying the Bernstein inequality conditionally, we obtain

$$
\sum_{n=1}^{\infty} P_{n}(\varepsilon) \leqslant 2 \sum_{n=1}^{\infty} e^{-W_{\varepsilon} m_{n} / t_{n}}
$$

where $W_{\varepsilon}=3 \varepsilon^{2} /[6 V+4 \varepsilon]$. Since the asymptotic equality $\left[x_{n} \log x_{n}\right] /\left[t_{n} \log t_{n}\right] \sim \alpha^{-\alpha} r_{n} \rightarrow$ $\infty$ holds for $x_{n} \equiv n^{1 / \alpha} / \log n$, we must have $s_{n}:=n^{1 / \alpha} /\left[t_{n} \log n\right]=x_{n} / t_{n} \rightarrow \infty$. Thus $m_{n} / t_{n}=w_{n} \log n$, where $w_{n}=m_{n} s_{n} / n^{1 / \alpha} \rightarrow \infty$ by the condition on $\left\{m_{n}\right\}$, and hence $\sum_{n=1}^{\infty} P_{n}(\varepsilon)<\infty$ almost surely for every $\varepsilon>0$.

(ii) By Lemma 4.7 there exists a positive sequence $\left\{r_{n}\right\}_{n \in \mathbb{N}}$ such that $r_{n} \uparrow \infty, n / r_{n} \uparrow \infty$ and $\sum_{n=1}^{\infty} \mathbb{P}\left\{|X|^{\alpha}>\frac{n}{r_{n}}\right\}<\infty$. If we redefine the truncating sequence as $t_{n}:=\left(n / r_{n}\right)^{1 / \alpha}, n \in \mathbb{N}$, then the proof of Theorem 2.1 in [1], i.e., a simplified version of the proof above, again 
gives that

$$
\sum_{n=1}^{\infty} P_{n}(\varepsilon) \leqslant 2 \sum_{n=1}^{\infty} e^{-W_{\varepsilon} m_{n} / t_{n}}
$$

almost surely for each $\varepsilon>0$. The last series is almost surely finite because $m_{n} / t_{n}=y_{n} \log n$ and $y_{n}=r_{n}^{1 / \alpha} m_{n} /\left[n^{1 / \alpha} \log n\right] \rightarrow \infty$ by the present condition on $\left\{m_{n}\right\}$.

(iii) Now using $t_{n}:=t^{-1} \log n$ for any fixed $t \in\left(0, t_{*}\right), n \geqslant 2$, and $t_{1}:=t_{2}$, we have

$$
\sum_{n=1}^{\infty} \mathbb{P}\left\{|X|>t_{n}\right\}=\sum_{n=1}^{\infty} \mathbb{P}\left\{e^{t|X|}>n\right\}<\infty
$$

by Lemma 4.7, as applied with $Y=e^{t|X|}$ and $r(\cdot)=1$. Thus, with this $\left\{t_{n}\right\}$, the proof above and the condition on $m_{n}$ again yield

$$
\sum_{n=1}^{\infty} P_{n}(\varepsilon) \leqslant 2 \sum_{n=1}^{\infty} e^{-W_{\varepsilon} m_{n} / t_{n}}<\infty
$$

almost surely for each $\varepsilon>0$.

\section{Remarks and conjectures}

Let $\mathscr{I}_{n}(\alpha)$ be either $\mathscr{I}_{n}^{\Delta}(\alpha)$ or $\mathscr{I}_{n}^{\star}(\alpha)$ from Theorems 2.1 and 2.2. Since the series

$$
\sum_{n=1}^{\infty} n^{-\min (\alpha-2,(\alpha-3) K)}=\infty,
$$

for $\alpha \in[1,3]$ our present approach appears to be insufficient for the strong sparsity property that

$$
\sum_{n=1}^{\infty} \mathbb{P}\left\{\left|\mathscr{I}_{n}(\alpha) \cap\left(\cup_{j=n+1}^{\infty} \mathscr{I}_{j}(\alpha)\right)\right| \geqslant K\right\}<\infty
$$

for some constant $K=K(\alpha)>0$; $c f$. the proofs of Theorem 2.1 and Lemma 2.12. (The referee has kindly pointed out that, by a reasoning more elaborate than ours the term $\mathbb{P}\left\{A_{n}^{c}\right\}=O\left(n^{-(\alpha-2)}\right)$ can be avoided in the proof of Theorem 2.1. Unfortunately, the resulting bound on $\mathbb{P}\left\{\left|\mathscr{I}_{n}^{\Delta}(\alpha) \cap\left(\cup_{j=n+1}^{\infty} \mathscr{I}_{j}^{\Delta}(\alpha)\right)\right| \geqslant K\right\}$ still remains $O\left(n^{-(\alpha-3) K}\right)$, and hence we have kept our simpler proof.) However, we conjecture that a weaker sparsity property still holds for $\alpha \in(2,3]$ : it is that

$$
\sum_{n=1}^{\infty} \mathbb{P}\left\{\left|\mathscr{I}_{n}(\alpha) \cap\left(\cup_{j=n+1}^{\infty} \mathscr{I}_{j}(\alpha)\right)\right| \geqslant K_{\alpha} n^{3-\alpha}\right\}<\infty
$$

for some constant $K_{\alpha}>0$. If this were true, then we could follow the present proof to show in the necessity direction that convergence in Theorem 3.1 for $\alpha \in(2,3]$ also implies $\mathbb{E}\left(|X|^{\beta}\right)<\infty$ for all $\beta \in[1,2)$. Similarly, Theorem 4.2 would then become true for $\alpha>2$ and the improved conclusion that $\mathbb{E}\left(|X|^{\beta}\right)<\infty$ for all $\beta \in\left[1,1+\frac{\alpha}{2}\right)$.

We also believe that for $\alpha>2$ the conjecture above is a special case of an even more challenging conjecture: the distribution of $\left|\mathscr{I}_{n}(\alpha) \cap\left(\cup_{j=n+1}^{\infty} \mathscr{J}_{j}(\alpha)\right)\right|$ is approximately Poisson 
with mean $C_{\alpha} / n^{\alpha-3}$, where the likely value of the constant $C_{\alpha}$ is $1 /(\alpha-2)$. The main difficulty is the inhomogeneity of the generated graph $G\left(\cup_{j=1}^{\infty} \mathscr{I}_{j}(\alpha)\right)$.

Even if the first conjecture was true, however, it would still leave the gap $\alpha \in[1,2]$ in Theorem 3.1. Concerning bootstrap means, on the basis of Theorem 4.4 it appears to be safe to conjecture for any $\alpha \geqslant 1$ that if $\mu_{n}^{\Delta}(\alpha) \stackrel{\text { a.s. }}{\longrightarrow} \mu$ or $\mu_{n}^{\star}(\alpha) \stackrel{\text { a.s. }}{\longrightarrow} \mu$, in the notation of Theorem 4.2, then $\mathbb{E}\left(|X|^{\alpha}\right)<\infty$. Theorem 4.2 and even its possibly improved form coming from the conjecture above for $\alpha>2$ are quite far from this. And then we still would not know whether or not the logarithmic factors in Theorem 4.4 are necessary.

The case of the naive bootstrap $m_{n} \equiv n$ is the most interesting special case: if $\mu_{n}^{\Delta}(1) \stackrel{\text { a.s. }}{\longrightarrow} \mu$ or $\mu_{n}^{\star}(1) \stackrel{\text { a.s. }}{\longrightarrow} \mu$, does it follow that $\mathbb{E}\left(|X| \log ^{+}|X|\right)<\infty$ ? Or, alternatively, is the finiteness of $\mathbb{E}(|X|)$ sufficient for $\mu_{n}^{\Delta}(1) \stackrel{\text { a.s. }}{\longrightarrow} \mu$ and $\mu_{n}^{\star}(1) \stackrel{\text { a.s. }}{\longrightarrow} \mu$, or for $\mu_{n}(n) \stackrel{\text { a.s. }}{\longrightarrow} \mu$ universally? As a more precise statement of Theorem 2.3, in this 'naive' case Theorem 2.4 suggests using the decomposition

$$
\sum_{j=1}^{n} X_{\left\lfloor n U_{j}\right\rfloor}=\sum_{j \in \mathscr{I}_{n}}^{n} X_{j}=\sum_{k=1}^{n} k \sum_{j \in R_{k}\left[\mathscr{Y}_{n}\right]} X_{j},
$$

in which the inner sums over disjoint sets become asymptotically independent. The main difficulty with this is the relationship between the random sets $R_{k}\left[\mathscr{I}_{n}\right]$ for different $n$.

\section{Acknowledgements}

We thank Professor Y. S. Chow for his interest in this work, Professor C. Matrán for clarifying a point in [1], Professor E. Arenal for spotting an inaccuracy in the first version, Professor C.-H. Zhang for simplifying our original proof of Theorem 2.4, and the referee for offering insightful comments.

\section{References}

[1] Arenal-Gutiérrez, E., Matrán, C. and Cuesta-Albertos, J. A. (1996) On the unconditional strong law of large numbers for the bootstrap mean. Statist. Probab. Lett. 27 49-60.

[2] Athreya, K. B. (1983) Strong law for the bootstrap. Statist. Probab. Lett. 1 147-150.

[3] Athreya, K. B., Ghosh, M., Low, L. Y. and Sen, P. K. (1984) Laws of large numbers for bootstrapped U-statistics. J. Statist. Plann. Inference 9 185-194.

[4] Barbour, A. D., Holst, L. and Janson, S. (1992) Poisson Approximation, Oxford University Press, Oxford.

[5] Baum, L. E. and Katz, M. (1965) Convergence rates in the law of large numbers. Trans. Amer. Math. Soc. 120 108-123.

[6] Bickel, P. J. and Freedman, D. A. (1981) Some asymptotic theory for the bootstrap. Ann. Statist. 9 1196-1217.

[7] Bollobás, B. (1979) Graph Theory: An Introductory Course, Springer, New York.

[8] Bollobás, B. (1985) Random Graphs, Academic Press, London.

[9] Chow, Y. S. and Teicher, H. (1988) Probability Theory, 2nd edn, Springer, New York.

[10] Csörgő, S. (1992). On the law of large numbers for the bootstrap mean. Statist. Probab. Lett. 14 1-7.

[11] Efron, B. (1979) Bootstrap methods: another look at the jackknife. Ann. Statist. 7 1-26.

[12] Erdős, P. (1949) On a theorem of Hsu and Robbins. Ann. Math. Statist. 20 286-291. 
[13] Erdős, P. (1950) Remark on my paper 'On a theorem of Hsu and Robbins'. Ann. Math. Statist. 21138.

[14] Etemadi, N. (1981) An elementary proof of the strong law of large numbers. Z. Wahrscheinlichkeitstheorie verw. Gebiete 55 119-122.

[15] Hsu, P. L. and Robbins, H. (1947) Complete convergence and the law of large numbers. Proc. Nat. Acad. Sci. USA 33 25-31.

[16] $\mathrm{Hu}, \mathrm{T}$-C. (1991) Strong consistencies of the bootstrap moments. Internat. J. Math. Math. Sci. 14 797-802.

[17] Hu, T.-C. and Taylor, R. L. (1997) On the strong law for arrays and for the bootstrap mean and variance. Internat. J. Math. Math. Sci. 20 375-382.

[18] Kolchin, V. F., Sevast'yanov, B. A. and Chistyakov, V. P. (1978) Random Allocations, Wiley, New York.

[19] Markov, A. A. (1912) Wahrscheinlichkeitsrechnung, Teubner, Leipzig.

[20] Mikosch, T. (1994) Almost sure convergence of bootstrapped means and U-statistics. J. Statist. Plann. Inference 41 1-19.

[21] Shiryaev, A. N. (1995) Probability, Springer, New York.

[22] Steinebach, J. (1980) Large Deviation Probabilities and Some Related Topics, Vol. 28 of Carleton Mathematical Lecture Notes, Carleton University, Ottawa. 